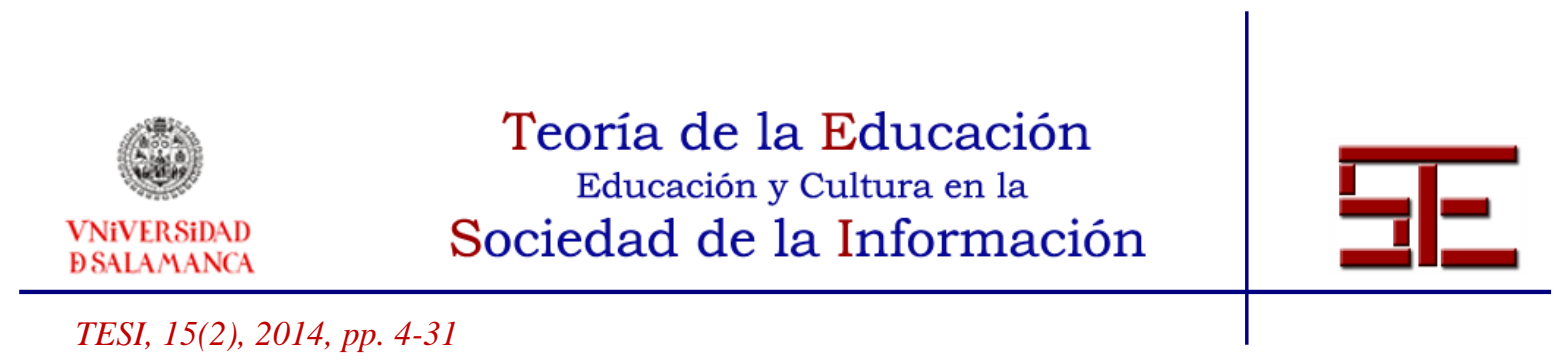

\title{
DIMENSIONES PARA EL DISEÑO Y CATALOGACIÓN DE OBJETOS DE APRENDIZAJE EN BASE A COMPETENCIAS INFORMACIONALES
}

Resumen: Una de las competencias básicas a desarrollar en España desde la educación primaria es "tratamiento de la información y competencia digital", sin embargo, a nivel universitario se observa que en muchos casos los estudiantes no cuentan con las habilidades necesarias para una adecuada gestión de la información, por tanto, se requiere de una estrategia que ayude a potenciar el desarrollo de competencias informacionales que les permitan desenvolverse adecuadamente en las diversas tareas que demanda una formación profesional. A través de este estudio se presenta una propuesta que considera la creación y clasificación de objetos de aprendizaje, para el desarrollo de competencias informacionales, basada en tres dimensiones: "4.1. Búsqueda, selección, almacenamiento y registro de información", "4.2. Organización, tratamiento y presentación de la información” y "4.3. Comunicación de la información”. Sobre esta base, se han creado cuatro objetos de aprendizaje, los cuales han sido evaluados por parte de estudiantes y docentes. A través de esta comunicación se presentan los resultados obtenidos, además de una propuesta de adaptación de metadatos Dublin Core para la descripción de los OA en base a competencias informacionales a través del repositorio documental de la Universidad de Salamanca GREDOS (gredos.usal.es).

Palabras clave: Objetos de Aprendizaje; metadatos; competencias informacionales.

\begin{tabular}{|c|c|c|}
\hline & Erla Mariela Morales Morgado y & $\underline{T}$ \\
\hline 4 & Rosalynn Argelia Campos Ortuño & 4 \\
\hline
\end{tabular}




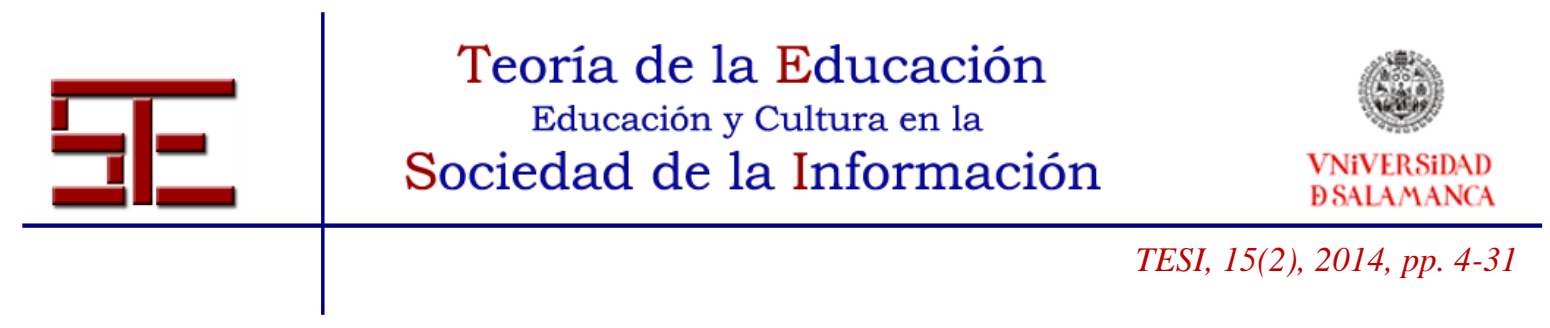

\title{
DIMENSIONS FOR LEARNIGN OBJECTS DESIGN AND MAPPING, BASED ON INFORMATION COMPETENCIES
}

\begin{abstract}
One of the basic competencies to develop in Spain from primary education is "information processing and digital competence", however, at the university level is observed that in many cases students do not have the necessary skills for proper management information, therefore it is needed a strategy to help the development of information skills that aim them deal appropriately with the various tasks required for their professional training. Through this study we suggest a proposal that considers the creation and object classification learning for skills development related with information competencies based on three dimensions: "4.1.Search for, selection, storage and recording of information"; "4.2. Organization, processing and presentation of information" and "4.3.Communication of information". On this basis, we have set up four learning objects, which have been evaluated by students and teachers. Through this communication the results are presented, along with a proposal to adjust Dublin Core metadata for describing LOs based on informational competencies through institutional repository of the University of Salamanca GREDOS (gredos.usal.es).
\end{abstract}

Key words: Learning Objects; metadata; information competencies.

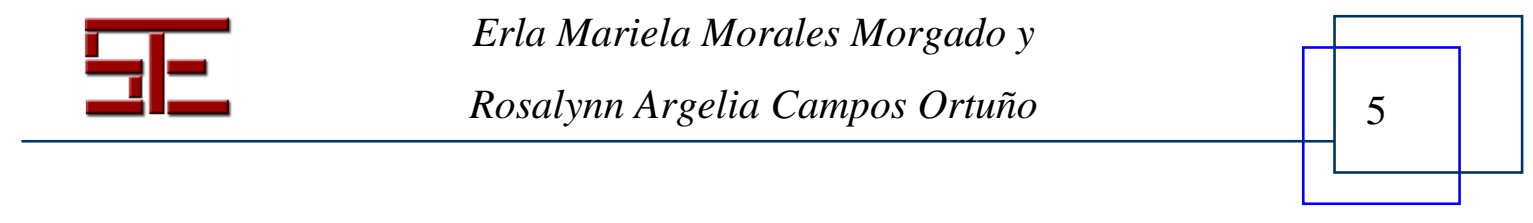




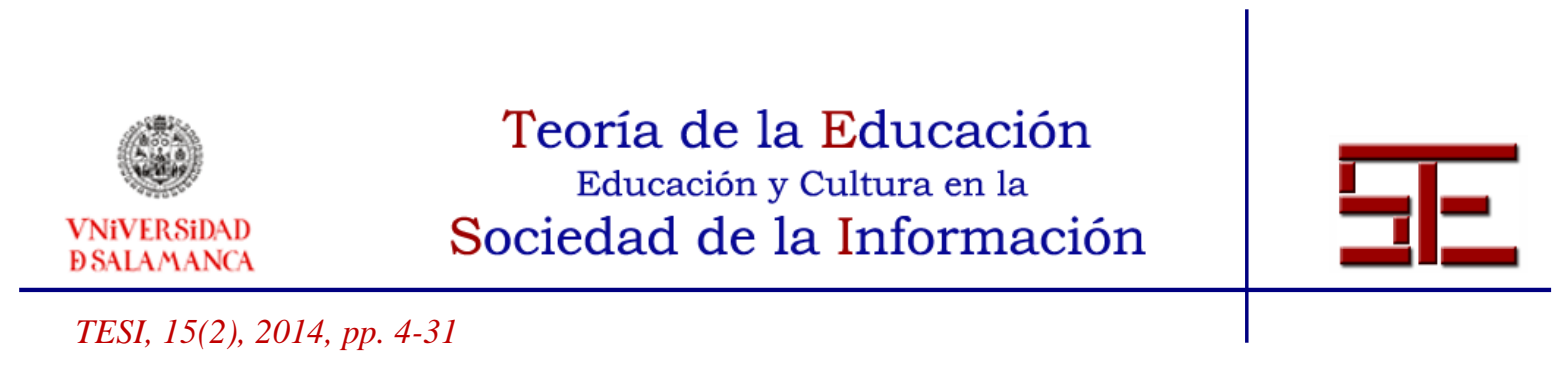

DIMENSIONES PARA EL DISEÑO Y CATALOGACIÓN DE OBJETOS DE APRENDIZAJE EN BASE A COMPETENCIAS INFORMACIONALES

Fecha de recepción: 15/02/2014; fecha de aceptación: 21/05/2014; fecha de publicación: 30/06/2014

Erla Mariela Morales Morgado

erla@usal.es

Universidad de Salamanca

Rosalynn Argelia Campos Ortuño

rosearcampos@gmail.com

Universidad de Salamanca

\section{1.- INTRODUCCIÓN}

Vivimos en una sociedad en donde el acceso a la tecnología forma parte de nuestra vida diaria, tal como señala Castells (1999), la era de la información está presente en nuestra economía, sociedad y cultura, por tanto no es de extrañar que la competencia digital sea considerada como una de las competencias básicas a desarrollar según establece el Parlamento Europeo y del Consejo de 18 de diciembre de 2006 (Diario Oficial de la Unión Europea, 2006) (González Briones, 2011). Entre las orientaciones generales que se proponen en dicho documento podemos destacar tres aspectos: la habilidad necesaria para acceder a servicios basados en Internet, buscarlos y utilizarlos; Producir, presentar y comprender información compleja, y saber cómo utilizar las TIC en apoyo del pensamiento crítico, la creatividad y la innovación.

En el contexto español, se ha establecido a través de los reales decretos de educación primaria $^{1}$ y secundaria ${ }^{2}$ el desarrollo de siete competencias básicas para la formación continua y permanente, conocida también como (lifelong learning) según la Commission of the European communities (2000) (Gaio, Neves y Gómez, 2010).

\footnotetext{
${ }^{1}$ España. Real Decreto-ley 1513/2006, de 07 de diciembre, por el que se establecen las enseñanzas mínimas de la Educación primaria. Boletín Oficial del Estado, 08 de diciembre de 2006, núm. 293, pp. 43053-43102.

2 España. Real Decreto-ley 1631/2006, de 29 de diciembre, por el que se establecen las enseñanzas mínimas de la Educación Secundaria Obligatoria. Boletín Oficial del Estado, 05 de enero de 2007, núm. 5, pp. 677-773.
}

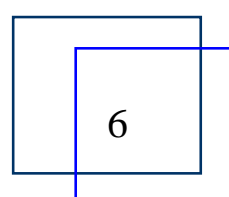

Erla Mariela Morales Morgado y

Rosalynn Argelia Campos Ortuño 


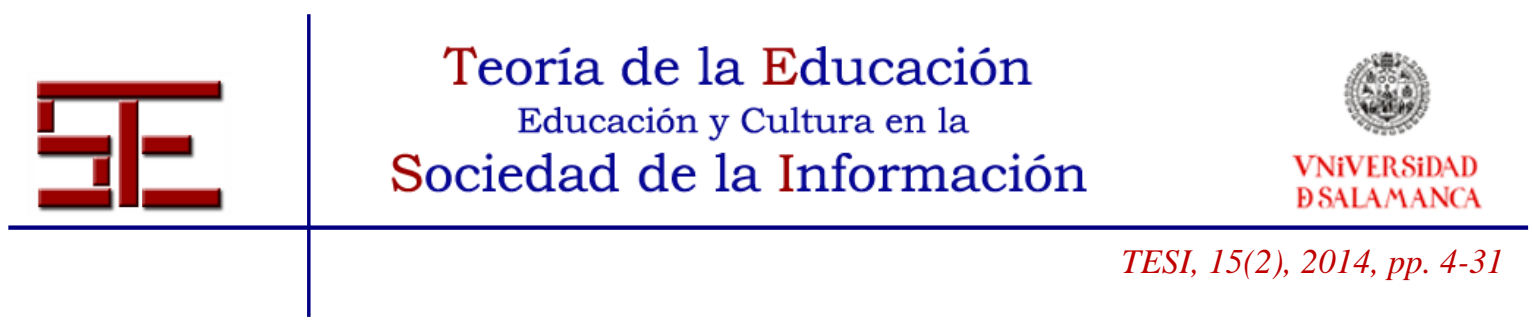

Una de las competencias básicas se denominada "competencia digital y tratamiento de la información", en donde se indican algunas orientaciones sobre las habilidades y destrezas que los estudiantes deben desarrollar, a través de las diversas áreas curriculares, tal como se indica en Duque et al. (2013).

Diversas investigaciones confirman la importancia y por qué formar en competencias informacionales en educación superior (Moreira, 2010) (CRUE-TIC y REBIUN, 2012), considerándolo como una materia absolutamente necesaria en la formación universitaria (Moreira, 2007). Sobre esta base, es posible encontrar algunas guías de buenas prácticas para el desarrollo de las competencias informacionales en las universidades españolas GRUPO ALFIN/REBIUN (2008); Gómez (2002); Gómez y Benito (2001).

$\mathrm{Al}$ respecto, ya se han realizado algunas propuestas a nivel de grado y posgrado en Universidades españolas, las cuales han considerado la colaboración de personal de bibliotecas y docentes, además de cursos de formación y elaboración de materiales, como el caso de la Universidad de Sevilla, Biblioteca (2009). Universitat Politècnica de Catalunya. Servei de biblioteques i documentació (2007) y la Universidad de La Laguna (Hernández, 2009ab).

A través de estas propuestas se evidencia que para poder aplicar una propuesta de formación en competencias informacionales se requiere de una política universitaria que permita impulsar diversas iniciativas que impliquen la participación y colaboración de personal administrativo que ayude en la capacitación, aunado al personal docente investigador que promueva el desarrollo de las habilidades y destrezas necesarias a través de las diversas actividades curriculares.

En el caso de la Universidad de Salamanca, los esfuerzos hasta ahora realizados no han sido suficientes para atender las demandas que los estudiantes requieren para desenvolverse adecuadamente en las diversas materias. La participación de personal de bibliotecas se limita a una sesión informativa sobre los diversos servicios que la universidad ofrece para la búsqueda de información, ya que no existe un plan de formación inicial a nivel institucional para estas cuestiones. Los temas relacionados con las nuevas tecnologías son tratados en asignaturas específicas a través de estudios de grado, licenciaturas y posgrado, sin embargo, la amplitud del programa de trabajo y el tiempo destinado no es suficiente para poder adquirir de una manera efectiva todas las habilidades y destrezas que requiere el proceso de búsqueda, selección, tratamiento y comunicación de la información.

Como señala Hernández \& Agustí (2011) “A pesar de que las habilidades informacionales son hoy auténticas competencias clave para el aprendizaje autónomo y para el aprendizaje a lo largo de la vida, para muchos estudiantes actuar como buscadores competentes es todavía un reto". Esto es debido a que la búsqueda en

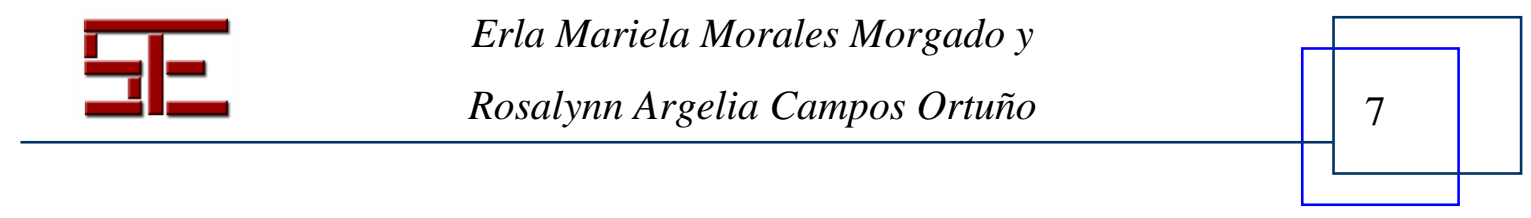




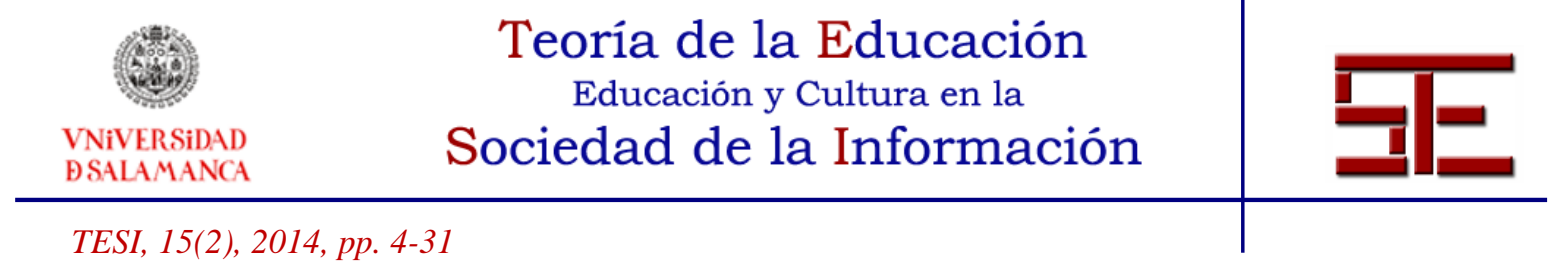

Internet es una tarea compleja y altamente dinámica, que depende de numerosas variables que se influencian recíprocamente.

En función de los lineamientos generales, es de suma importancia definir propuestas específicas en relación a "cómo" desarrollar la competencia informacional, la manera de abordar todos los aspectos que en la literatura nos indican el "qué" es lo que hace a los usuarios competentes en el manejo de la información.

Para atender a esta necesidad, surge el proyecto DIRED (Divulgación de Recursos Educativos a través de la Red) (Morales et al., 2013b), un proyecto cuyo objetivo es la investigación, para el diseño, desarrollo y difusión de recursos educativos que puedan ser de interés para complementar el desarrollo académico en la Universidad de Salamanca (diarium.usal.es/linglingyang).

Dentro de los recursos educativos, se encuentra el desarrollo de objetos de aprendizaje, los cuales según sus características, pueden ser diseñados para objetivos de enseñanza específicos, ya sea de tipo conceptual, procedimental o actitudinal, ingredientes clave para el desarrollo de competencias (Morales et al., 2011). Sobre esta base, se han diseñado OAs basados en la competencia informacional, de manera de facilitar a los estudiantes recursos con objetivos específicos, acompañados de contenidos y actividades para su logro.

El primer paso antes de su diseño ha sido definir las dimensiones y sub-competencias a desarrollar, de manera de atender a las necesidades de búsqueda, selección, tratamiento y comunicación de la información. El segundo apartado presenta la definición de tres dimensiones, además de los criterios, habilidades y destrezas a desarrollar en cada una de ellas. En base a estas definiciones, se procedió a la definición de cuatro temas que se consideraron prioritarios para complementar la formación de los estudiantes en relación a la búsqueda de información, cuyo diseño y contenido se explica en el tercer apartado.

Los OA fueron aplicados a estudiantes de primer a cuarto año de la carrera de Magisterio en Educación Infantil y Primaria, quienes valoraron los recursos en función de su adecuación a los objetivos, contenidos y actividades de enseñanza, además de dar a conocer su grado de satisfacción con respecto a los recursos. Por otra parte, los OA fueron valorados por un grupo de docentes que forman parte de un proyecto de innovación relacionado a las actividades de DIRED, con la finalidad de realizar las mejoras necesarias antes de su publicación en el repositorio institucional GREDOS, en donde van a ser alojados para el acceso por parte de cualquier miembro de la comunidad universitaria. El cuarto apartado muestra los resultados obtenidos por parte de la valoración de estudiantes y profesores y, finalmente, el quinto apartado presenta una propuesta para alojar los OA en colecciones específicas que permitan catalogarlos por según competencias informacionales a través del repositorio institucional GREDOS.

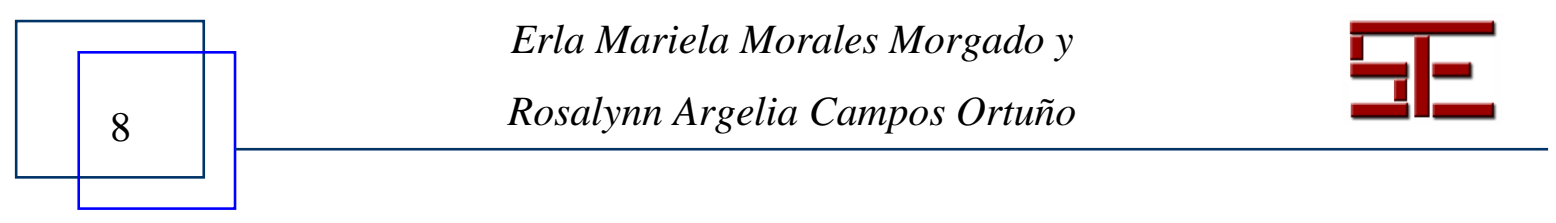




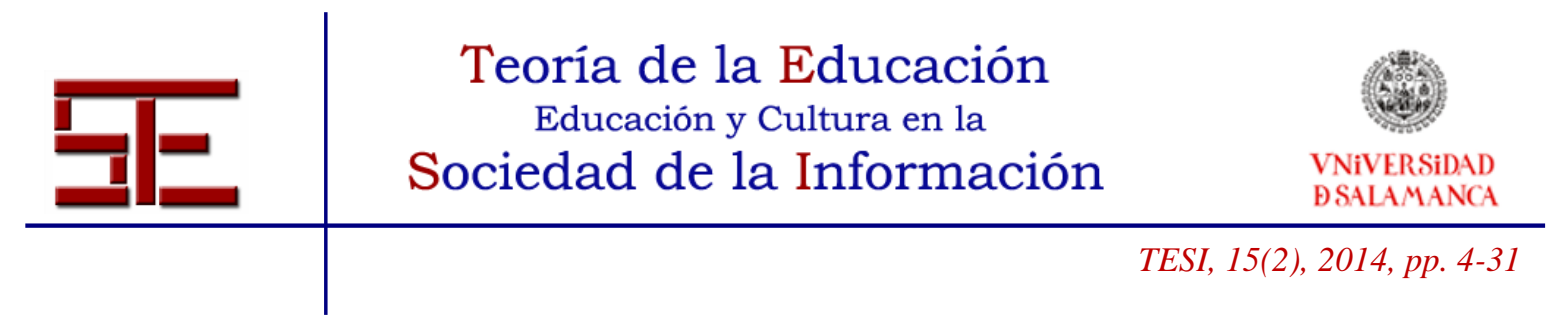

\section{2.- DIMENSIONES PARA LA CLASIFICACIÓN DE OBJETOS DE APRENDIZAJE BASADOS EN COMPETENCIA INFORMACIONAL}

Tal como señala el Manual para la formación en competencias informáticas e informacionales (CI2) de CRUE-TIC \& REBIUN (2013): "La vigente estructuración de las enseñanzas y títulos universitarios en España incorpora en los planes de estudios de los títulos de grado diferentes competencias transversales, entre las que destacan el uso de herramientas informáticas y la habilidad en la búsqueda, el análisis y la gestión de la información", capacidades consideradas a ser desarrolladas para toda la vida, las cuales deben ser consideradas como estrategia educativa en el ámbito universitario.

Ante esta necesidad surgen algunas orientaciones sobre la formación en competencias informacionales, dirigidas a los profesionales, encargados de diseñar e implementar programas formativos en estas áreas. Entre ellos destacan el proceso de búsqueda de información, que conlleva la detección de la necesidad de información y una adecuada selección en fuentes adecuadas para atender esa necesidad. Complementario a lo anterior es la evaluación de la información, para lo cual hay que tener criterios que ayuden a discernir la adecuación y validez de la información, con una actitud crítica y reflexiva sobre la misma. Una vez obtenida la información es importante conocer herramientas para organizarla y comunicarla, como, por ejemplo, los gestores de contenidos y referencias, así como también criterios para usar éticamente la información, lo cual requiere el conocimiento del concepto de plagio, licencias y derechos de autor.

El desarrollo de esta competencia a nivel autonómico se ve reflejado en algunas propuestas como la del Gobierno Vasco( http://ediagnostikoak.net/edweb/cas/materialesinformativos/ED_marko_teorikoak/Marc o_competencia_digital_cas.pdf), en donde se proponen dimensiones, sub-competencias, indicadores de cada competencia y gradación para $4^{\circ}$ curso de primaria y $2^{\circ}$ curso de la ESO.

Actualmente podemos encontrar algunas referencias que nos indican las capacidades que los usuarios deben desarrollar para una adecuada gestión de las TIC y ejemplos de actividades para su logro (Vivancos, 2008; Duque et al., 2013; Cárdenas, 2010).

Considerando la información disponible para atender al desarrollo de la competencia digital, es necesario definir, de forma concreta, las sub-competencias específicas a desarrollar, indicando además los respectivos criterios, descriptores y habilidades y destrezas relacionadas. Sobre esta base, se definen las siguientes tres dimensiones:

4.1.Búsqueda, selección, almacenamiento y registro de información;

4.2. Organización, tratamiento y presentación de la información;

4.3. Comunicación de la información.

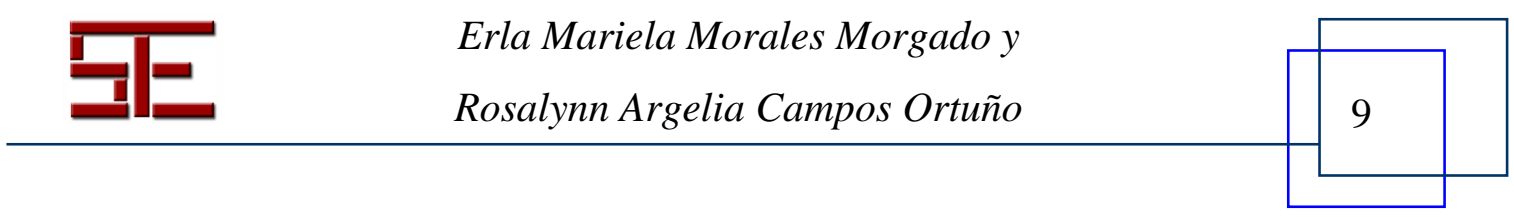




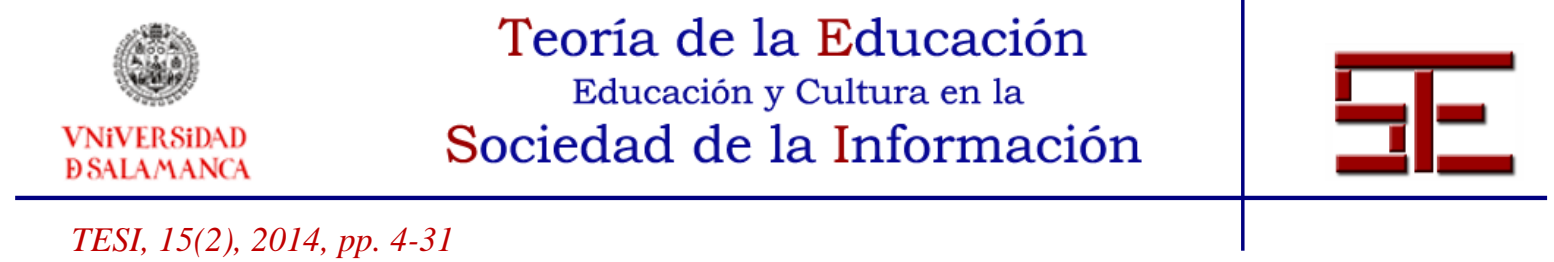

A través de las Tablas 1, 2 y 3, se presenta una propuesta basada en tres dimensiones, cada una presenta: Criterios que permitan valorar el conocimiento adquirido, Descriptores que especifiquen de forma más concreta el tipo de actividades que los usuarios deben ser capaces de realizar y, finalmente, las habilidades y destrezas que debe manejar para desenvolverse adecuadamente en cada dimensión.

Tabla 1. Dimensión: 4.1. Búsqueda, selección, almacenamiento y registro de información de la Competencia Digital

\begin{tabular}{|c|c|}
\hline \multicolumn{2}{|c|}{ Dimensión 4.1: Búsqueda, selección, almacenamiento y registro de información } \\
\hline Criterio & $\begin{array}{l}\text { Conoce las características de diferentes tipos de fuentes de } \\
\text { información. } \\
\text { Reconoce los tipos de recursos que caracterizan a las diversas fuentes. } \\
\text { Diferencia y valora en base a criterios el tipo de información que } \\
\text { contienen las fuentes. } \\
\text { Utiliza dispositivos virtuales de almacenamiento. } \\
\text { Conoce formas de registrar y organizar información de interés. }\end{array}$ \\
\hline $\begin{array}{l}\text { Descripto } \\
\text { res }\end{array}$ & $\begin{array}{l}\text { 4.1.1. Accede a la información a través de herramientas y estrategias } \\
\text { específicas. } \\
\text { 4.1.2. Conoce fuentes fiables de información. } \\
\text { 4.1.3. Valora de forma crítica y sistemática la pertinencia de la } \\
\text { información. } \\
\text { 4.1.4. Almacena y registra información en diversas fuentes. }\end{array}$ \\
\hline $\begin{array}{l}\text { Habilidad } \\
\text { es y } \\
\text { destrezas }\end{array}$ & $\begin{array}{l}\text { Manejo de diversos tipos de Buscadores, utilizando operadores que } \\
\text { faciliten la búsqueda de información en varios soportes. } \\
\text { Valoración acerca de la confiabilidad y adecuación de la información } \\
\text { obtenida en diversas fuentes. } \\
\text { Uso de herramientas y técnicas para registrar y organizar la } \\
\text { información recopilada. Citación correcta de referencias bibliográficas. }\end{array}$ \\
\hline
\end{tabular}

La Dimensión 4.1: Búsqueda, selección, almacenamiento y registro de información ${ }^{3}$, hace referencia a las habilidades básicas necesarias para buscar y seleccionar la

\footnotetext{
${ }^{3}$ Las dimensiones comienzan con el número 4, ya que la competencia digital se encuentra con esa numeración dentro de las competencias clave a desarrollar según el Parlamento Europeo. Se deja así
}

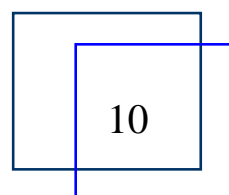

Erla Mariela Morales Morgado y

Rosalynn Argelia Campos Ortuño 


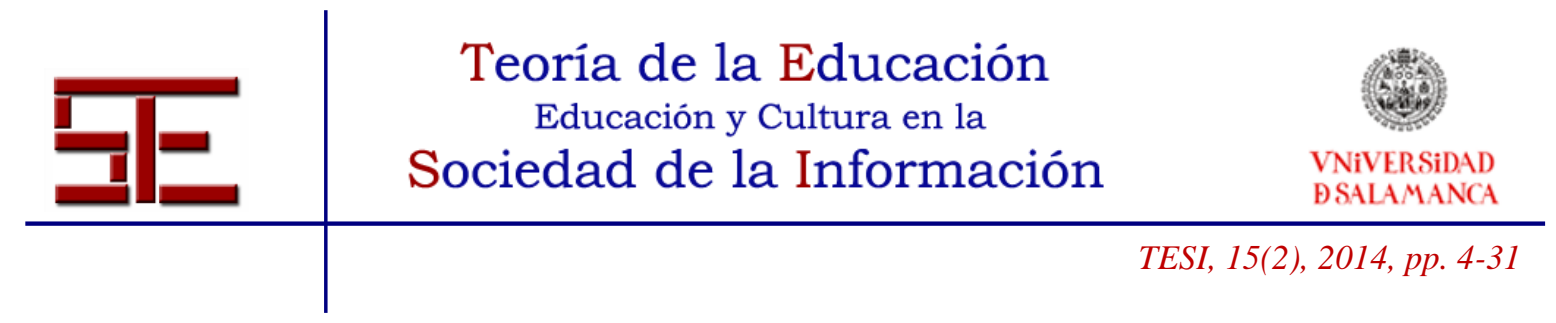

información de manera eficiente, lo cual requiere el conocimiento de diversos tipos de buscadores, opciones de búsqueda personalizada y especializada, fuentes de información primaria, secundaria y terciaria, bases de datos de áreas específicas, etc.

Además de aprender a buscar de forma adecuada, los usuarios deben aprender a reconocer los recursos obtenidos, valorar con criterios la calidad de la misma y, una vez seleccionada la información de interés, gestionarla a través de dispositivos virtuales que permitan su organización y almacenamiento.

Tabla 2. Dimensión 4.2: Organización, tratamiento y presentación de la información de la Competencia Digital

\begin{tabular}{|c|c|}
\hline \multicolumn{2}{|c|}{ Dimensión 4.2: Organización, tratamiento y presentación de la información } \\
\hline Criterio & $\begin{array}{l}\text { Manipula diversos tipos y códigos de información. } \\
\text { Conoce estrategias de organización de la información. } \\
\text { Expresa ideas claras a través de diversos códigos. }\end{array}$ \\
\hline $\begin{array}{l}\text { Descripto } \\
\text { res }\end{array}$ & $\begin{array}{l}\text { 4.2.1. Almacenamiento y registro de la información en diversas } \\
\text { fuentes. } \\
\text { 4.2.2. Maneja diversos tipos y códigos de información: textual, } \\
\text { numérica, icónica, gráfica, auditiva y audiovisual. } \\
\text { 4.2.3. Maneja herramientas para producir, presentar y comprender } \\
\text { información. } \\
\text { 4.2.4. Intercambia información. }\end{array}$ \\
\hline $\begin{array}{l}\text { Habilidad } \\
\text { es y } \\
\text { destrezas }\end{array}$ & $\begin{array}{l}\text { Manejo de diversos tipos de almacenamiento de información Gestores } \\
\text { de referencias bibliográficas. } \\
\text { Manejo de editores de imágenes, sonidos, videos, textos, etc. } \\
\text { Transformación de información en conocimiento. } \\
\text { Tratamiento de textos, hojas de cálculo, bases de datos. } \\
\text { Mapas conceptuales, programas para presentar la información. } \\
\text { Formatos para comprimir, descomprimir, reutilizar, etc. }\end{array}$ \\
\hline
\end{tabular}

La Tabla 2 indica la dimensión que está relacionada con el procesamiento de la información, es decir, establece las habilidades y destrezas que se deben desarrollar para

abierta la posibilidad de agregar materiales a la plataforma que formen parte de las otras competencias clave.

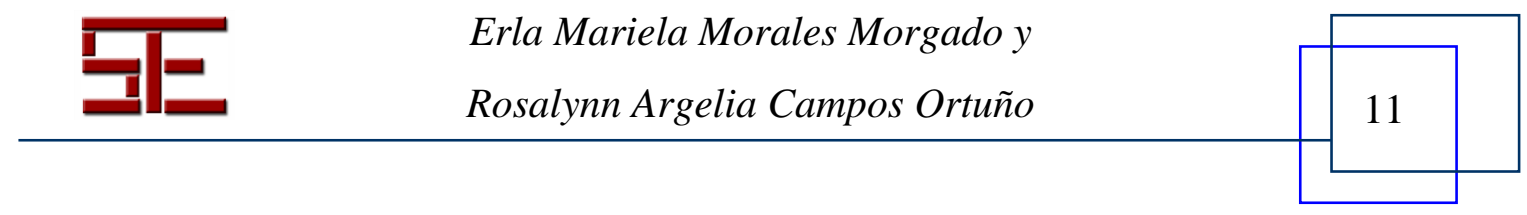




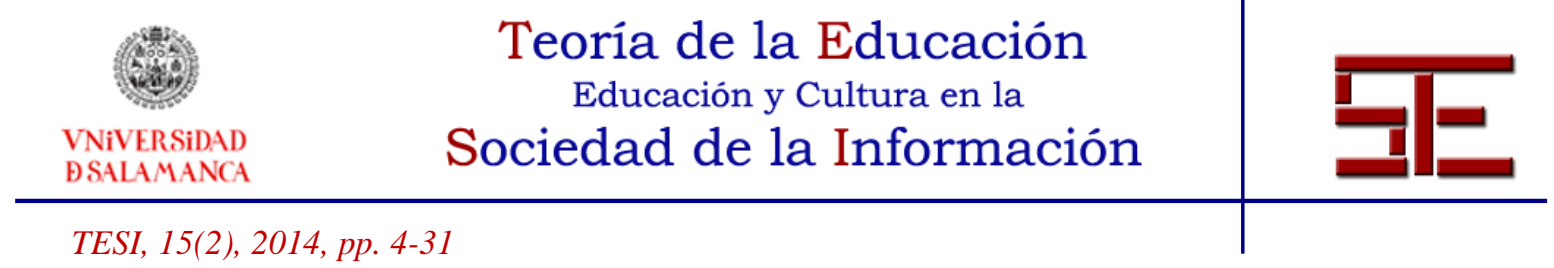

transformar la información en conocimiento, para lo cual es necesario conocer los diversos tipos de información, según el medio de comunicación al que pertenezca: textual, icónico, gráfico, audiovisual, etc. Programas para editar y organizar la información: mapas conceptuales, bases de datos, presentaciones, hojas de cálculo, etc.

Tabla 3. Dimensión 4.3: Comunicación de la información

\begin{tabular}{|c|c|}
\hline \multicolumn{2}{|c|}{ Dimensión 4.3: Comunicación de la información } \\
\hline Criterio & $\begin{array}{l}\text { Maneja herramientas para compartir y trabajar en colaboración. } \\
\text { Difunde información en diversos soportes y redes sociales. } \\
\text { Expresa sus ideas de forma crítica, creativa e innovadora. } \\
\text { Demuestra su identidad digital. }\end{array}$ \\
\hline $\begin{array}{l}\text { Descripto } \\
\text { res }\end{array}$ & $\begin{array}{l}\text { 4.3.1. Herramientas para compartir y trabajar en colaboración. } \\
\text { 4.3.2. Difusión de la información en diversos soportes. } \\
\text { 4.3.3. Desarrollo del pensamiento crítico, creatividad e innovación. } \\
\text { 4.3.4. Manifestación de identidad digital en redes sociales. }\end{array}$ \\
\hline $\begin{array}{l}\text { Habilidad } \\
\text { es y } \\
\text { destrezas }\end{array}$ & $\begin{array}{l}\text { Uso y gestión de herramientas de trabajo colaborativo. } \\
\text { Comunicación eficaz a través de diversas redes sociales. Ej. } \\
\text { GoogleDocs, Redes sociales: Blogs, wikis, micro-blogging, e- } \\
\text { Actividades, etc. } \\
\text { Adaptación de una identidad digital propia, para exponer sus ideas y } \\
\text { opiniones en sus actividades realizadas a través de la Red. }\end{array}$ \\
\hline
\end{tabular}

Finalmente, la tercera dimensión hace referencia a las capacidades necesarias para comunicar la información que se ha buscado, organizado y transformado en el propio conocimiento. Se destaca el manejo de herramientas para trabajar en colaboración y difusión de la información, desarrollando capacidades cognitivas que apunten a la elaboración de reflexiones personales que expresen ideas de forma crítica, creativa e innovadora.

Las tres dimensiones mencionadas para el desarrollo de la competencia digital constituyen una base orientativa para definir objetivos específicos de aprendizaje, diseñar actividades que permitan la consecución de dichos objetivos y seleccionar las herramientas más adecuadas para alcanzarlos.

\begin{tabular}{|l|l|l}
\hline 12 & $\begin{array}{l}\text { Erla Mariela Morales Morgado y } \\
\text { Rosalynn Argelia Campos Ortuño }\end{array}$ \\
\cline { 2 - 3 }
\end{tabular}




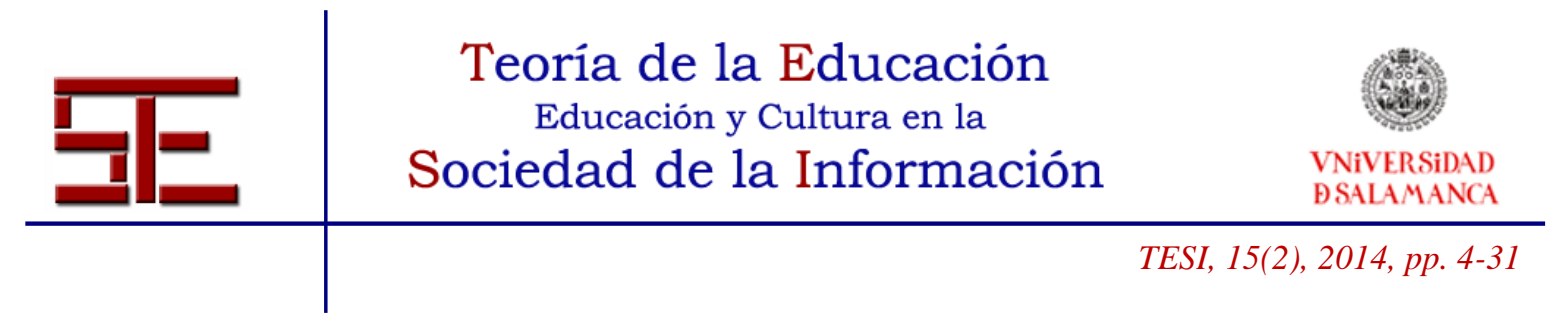

\section{3.- EJEMPLOS DE DISEÑO DE OBJETOS DE APRENDIZAJE BASADOS EN COMPETENCIA INFORMACIONAL}

La propuesta de clasificación explicada en el apartado anterior permite facilitar el diseño de los Objetos de Aprendizaje en cuanto a la selección y estructuración de contenidos. Es así como, considerando la dimensión 4.1. Búsqueda, selección, almacenamiento y registro de información, se han diseñado cuatro OA, los cuales han sido revisados y evaluados por expertos sobre su calidad y también por estudiantes para conocer su nivel de satisfacción en relación a la comprensión de la información.

Los OA diseñados han abordado los siguientes temas:

-Fuentes de información.

-Buscadores en la Red.

-Buscando en Google.

-Plagio académico y normas APA.
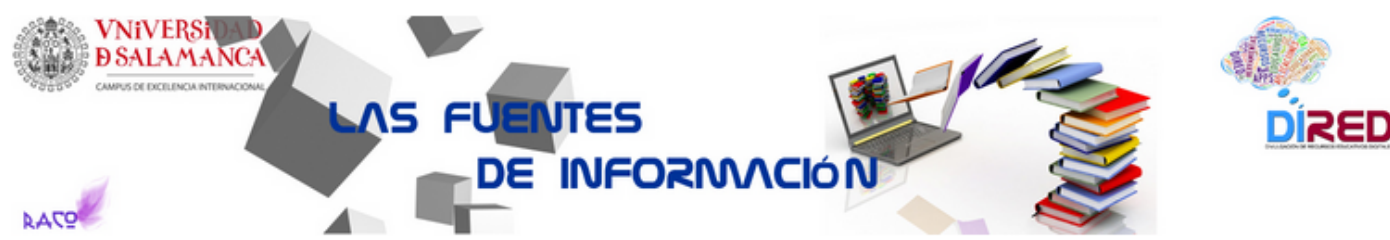

A través de este Objeto de Aprendizaje lograrás:

* Conocer las características de diferentes tipos de fuentes de información.

* Reconocer los tipos de recursos que caracterizan a las diversas fuentes.

* Diferenciar el tipo de información que contienen las fuentes.

Navegarás por los siguientes contenidos:

1. Tipos de fuentes, donde se hablará sobre:

- Primarias

- Secundarias

- Terciarias

2. Criterios para valorar la calidad de la información en las fuentes, donde se hablará sobre:
- Autores y editores de prestigio
- Estilos del documento
- Objetividad de la obra
- La información sea claras
- Fecha de publicación
- Usabilidad del sitio
- Argumentos

Figura 1. Objeto de Aprendizaje sobre fuentes de información.

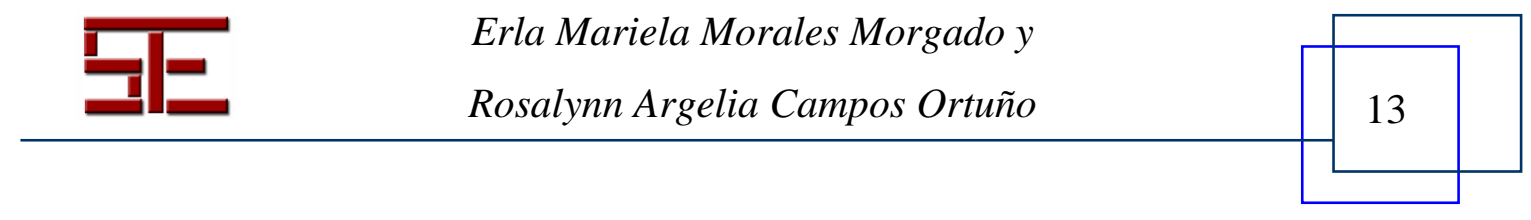




\begin{tabular}{c} 
Teoria de la Educación \\
Educación y Cultura en la \\
$\begin{array}{c}\text { VNiVERSIDAD } \\
\text { BSALAMANCA }\end{array}$ \\
\hline TESI, 15(2), 2014, pp. 4-31
\end{tabular}

El OA que presenta la figura 1 tiene como objetivo dar a conocer con ejemplos y casos los tipos de fuentes y sus características, además de diversos criterios a tener en cuenta para valorar la calidad de la información y seleccionar la que sea adecuada al problema de investigación inicial. El recurso contiene una actividad en donde debe entrar en una base de datos, buscar un tema específico y valorar la calidad de la información en función del autor y la fuente de información, se complementa con preguntas de reflexión.



Es cierto que INTERNET es una fuente de información inagotable e inmensa, y aunque muchos afirmen que allí está TODO ... No todo está bien, ACCESIBLE o

COMBROBADO... Por eso necesitamos a los BUSCADORES o MOTORES DE

BÚSQUEDA para encontrar la INFORMACIÓN que requerimos.

Con ayuda de este Objeto de Aprendizaje podrás:

- Conocer los diversos tipos de buscadores electrónicos existentes, reconocer sus funcionalidades y limitaciones para usar el tipo que mejor convenga a una demanda requerida.

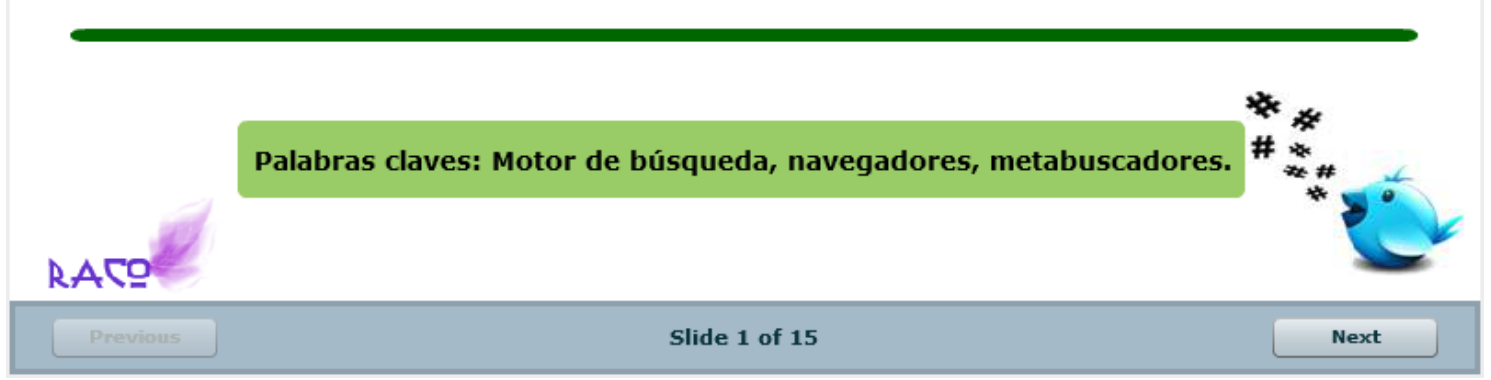

Figura 2. Objeto de Aprendizaje sobre buscadores en la Red.

El objetivo del OA que representa la figura 2 es ofrecer un material que amplíe la visión sobre las herramientas de búsqueda de información. Al consultar a los estudiantes qué herramientas utilizan para esta tarea, la mayoría ha contestado que acude a uno o dos buscadores populares: como Google, Explorer, Crome, etc. Esta cuestión evidencia un desconocimiento sobre conceptos y características, ventajas y desventajas de

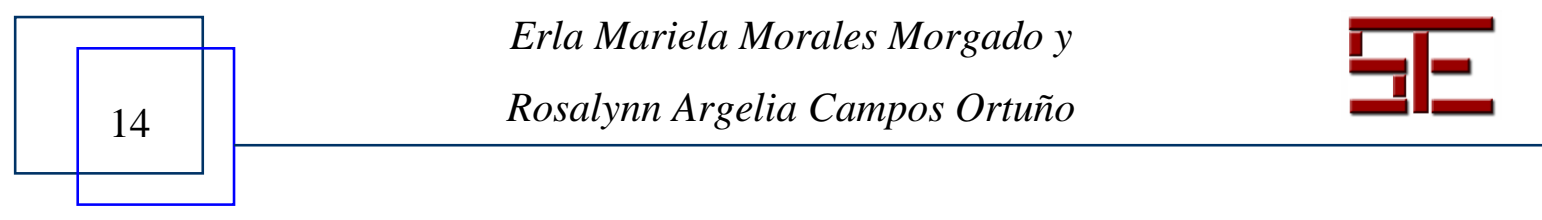




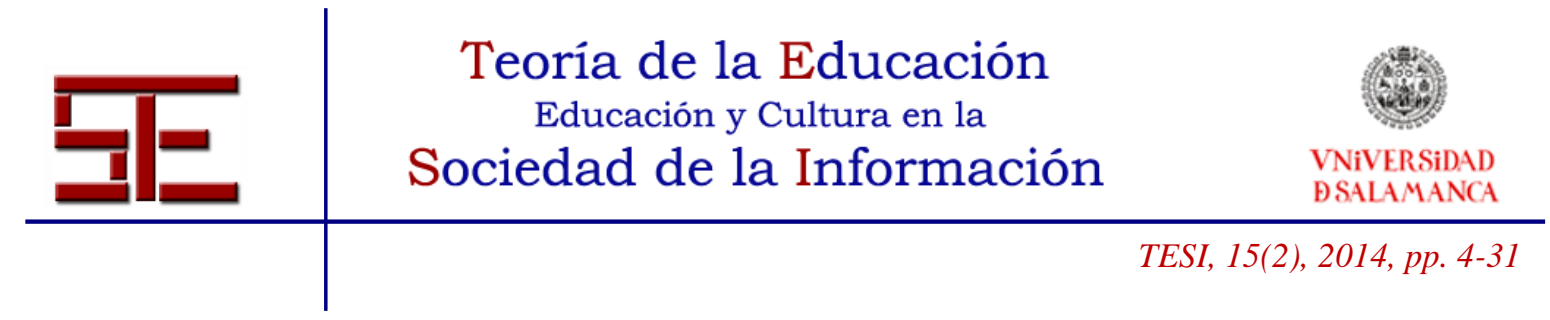

herramientas como los motores de búsqueda y los metabuscadores, además de cómo funcionan las búsquedas a través de la Red. Para dar a conocer estos conceptos, características, funcionalidades y limitaciones, se diseñó un OA sobre Buscadores en la Red.

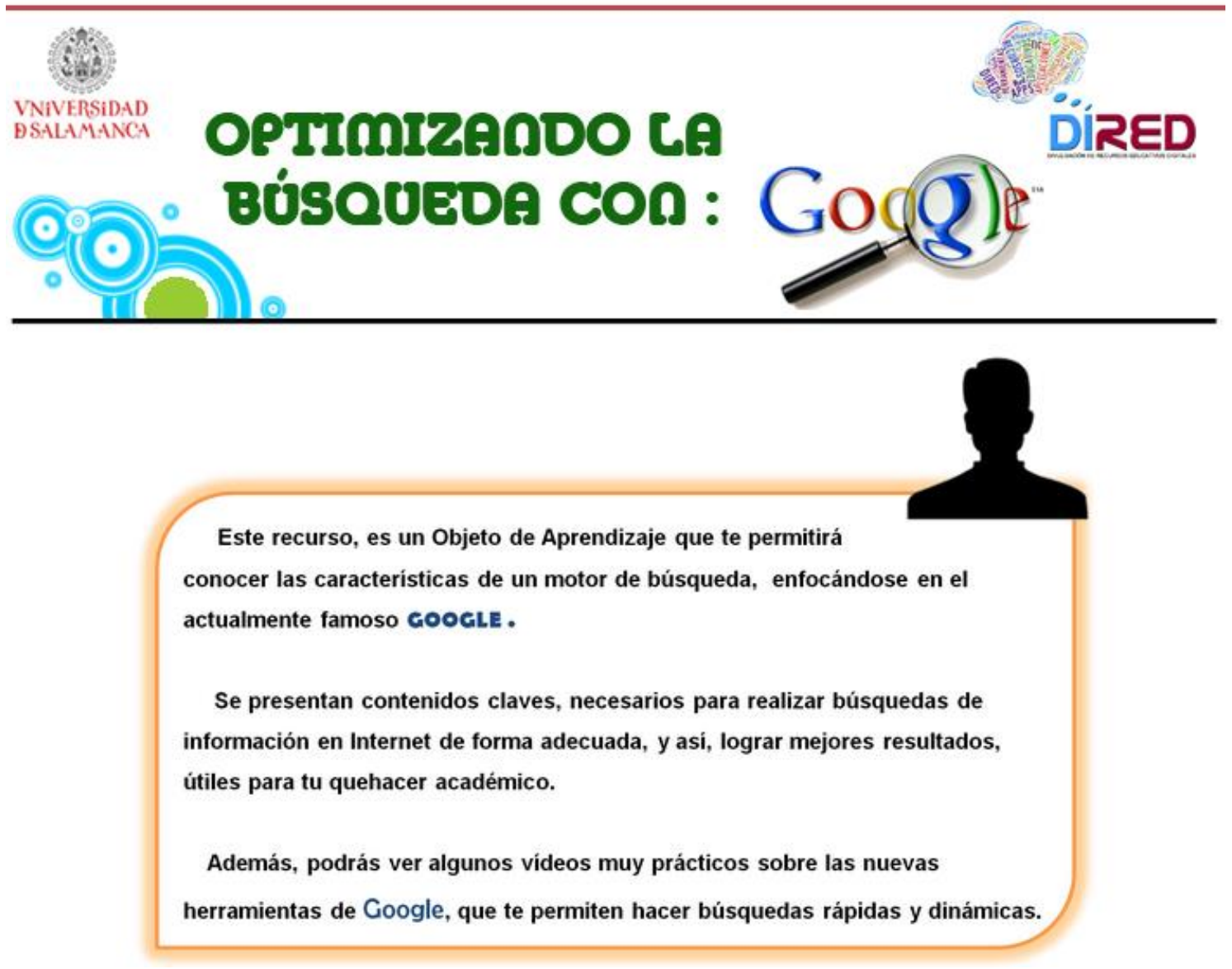

Figura 3. Optimizando la búsqueda con Google.

Google es sin duda uno de los buscadores más populares, sin embargo, al consultar a los estudiantes la forma de utilizarlo, la mayoría manifestó desconocer muchas de las posibilidades que esta herramienta ofrece, por esta razón se consideró oportuno crear un OA específico que ayude a conocer cómo realizar una búsqueda adecuada a través de operadores lógicos, opciones avanzadas, además de búsquedas según diversos formatos: filtros, voz, imágenes, avanzadas y segura. El OA presenta además herramientas asociadas como Google Académico y Google Books.

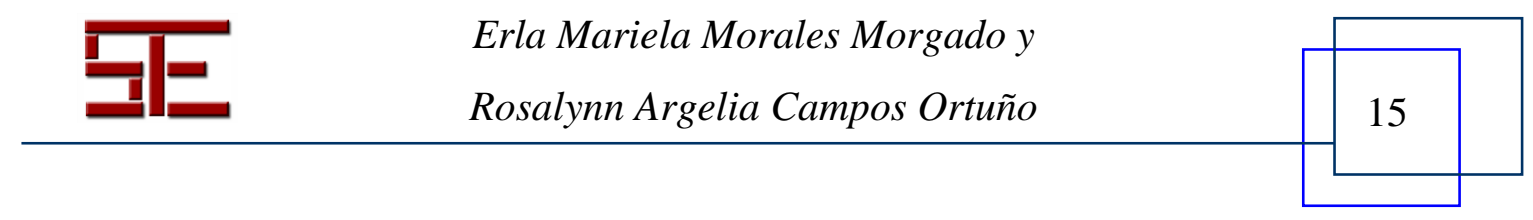



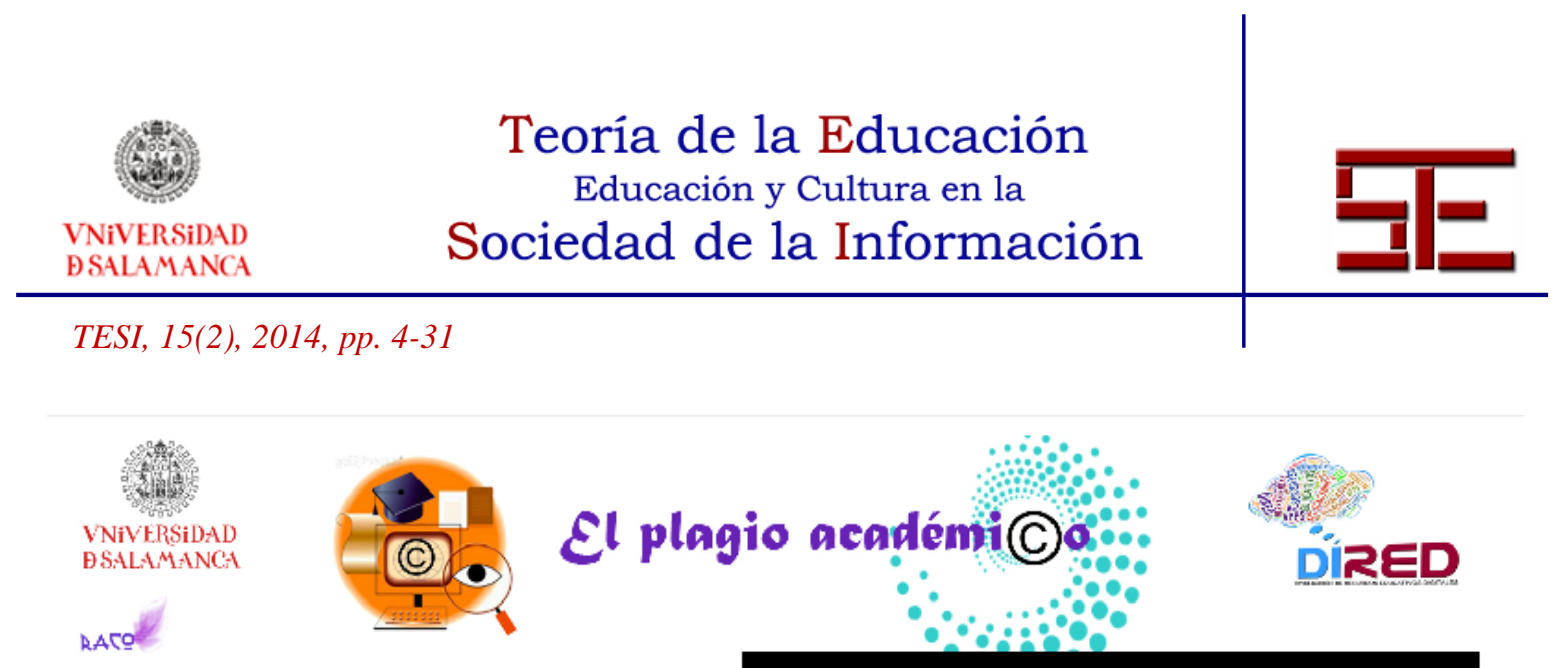

¿Cómo te sentirías si alguien ha copiado tu idea o argumento sin mencionar tu autoría?

PLAGIADO

Con ayuda de este Objeto de Aprendizaje podrás conocer el plagío, cómo identificarlo y evitarlo.
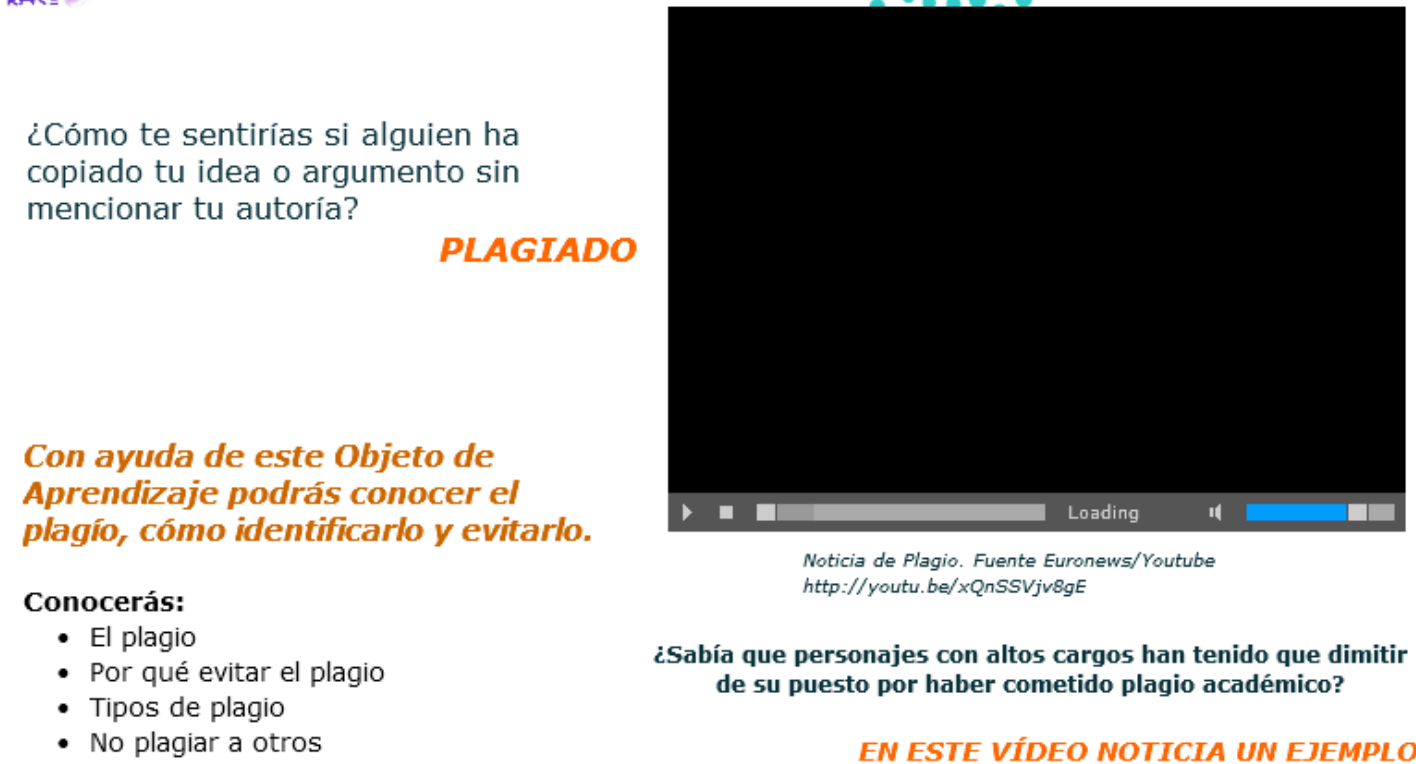

Noticia de Plagio. Fuente Euronews/Youtube

http://youtu. be/xQnSSVjv8gE

¿Sabía que personajes con altos cargos han tenido que dimitir de su puesto por haber cometido plagio académico?

EN ESTE VÍDEO NOTICIA UN EJEMPLO

Figura 4. Plagio académico y normas APA.

Finalmente, otro de los temas indiscutidos a abordar en uno de los OA es el uso ético de la información. Los estudiantes presentan bastante desconocimiento en relación al reconocimiento de un plagio, situación que se visualiza en diversas cuestiones, como es el no reconocimiento de la autoría al citar incorrectamente o no citar a un autor, así como también otro caso muy común que es el parafraseo.

Ante esta situación, se diseñó un OA llamado "Plagio académico", el cual intenta llamar la atención con elementos que le hagan comprender la importancia y gravedad que tiene el incurrir en este problema a través, como por ejemplo, de la visualización de un video, en donde una candidata a ministra de Educación debe dimitir en Alemania al conocerse que había plagiado su tesis doctoral. El OA presenta información sobre los derechos de autor o copyright que regulan los derechos morales y patrimoniales de la propiedad intelectual, además de información sobre el uso adecuado de las normas APA para citar correctamente la información. A modo de refuerzo, se presentan ejercicios en donde los estudiantes pueden poner a prueba los conocimientos teóricos adquiridos.

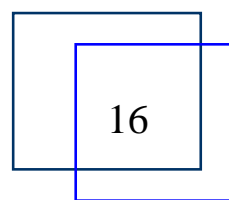

Erla Mariela Morales Morgado y

Rosalynn Argelia Campos Ortuño






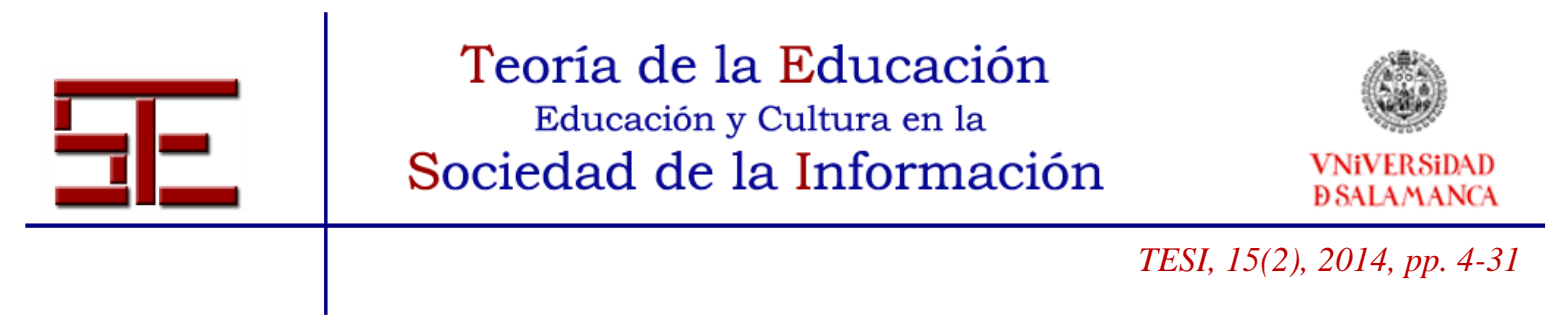

\section{4.- VALORACIÓN DE LOS OBJETOS DE APRENDIZAJE POR PARTE DE PROFESORES Y ESTUDIANTES}

La evaluación de objetos de aprendizaje ha sido nuestro objeto de estudio a través de diversas investigaciones, ya sea como unidades de aprendizaje (Morales et al., 2004); (Morales et al., 2005) a lo cual se ha sumado el diseño instruccional de calidad a través de un modelo ontológico (Morales, 2007b) que sugiere los elementos que deben contener los OA de diversos niveles de granularidad para garantizar una consistencia a nivel pedagógico. Posteriormente se definieron criterios pedagógicos, técnicos y también relativos a las características de los OA, para crear una herramienta de evaluación llamada HEODAR (Morales et al., 2008) que ha sido programada para poder integrarse como un módulo más de Moodle, con el fin de valorar OA en formato SCORM y arrojar de forma automática los aspectos mejor y peor valoraos de esa evaluación, para facilitar la mejora de los OA de manera continua (Muñoz et al., 2010ab).

La herramienta ha sido diseñada para la valoración por parte de expertos en aspectos pedagógicos y técnicos, de manera que valore un OA a la vez. Como nuestro objetivo era que los docentes evaluaran los OA en calidad de expertos, se diseñó u cuestionario basado en los criterios de HEODAR para evaluar los 4 OA a la vez. Para la evaluación de los OA por parte de estudiantes se aplicó un cuestionario más simple que se enfocara en el nivel de satisfacción con respecto al uso del recurso. A continuación se explicará en detalle en la exerencia de evaluación por parte de estudiantes y expertos.

\section{1.- Valoración por parte de estudiantes}

Los OA descritos en el apartado anterior, fueron trabajados y valorados por estudiantes de la Universidad de Salamanca, con la finalidad de realizar un análisis descriptivo sobre el nivel de satisfacción en el aprendizaje a través de los recursos. La muestra que participó en la revisión y valoración de los recursos estuvo comprendida por un total de 92 estudiantes de primero a cuarto año de magisterio en educación infantil y primaria. Los estudiantes revisaron uno de los cuatro recursos, realizando las actividades en ellos señaladas, a continuación contestaron un cuestionario que preguntaba sobre diversos aspectos educativos.

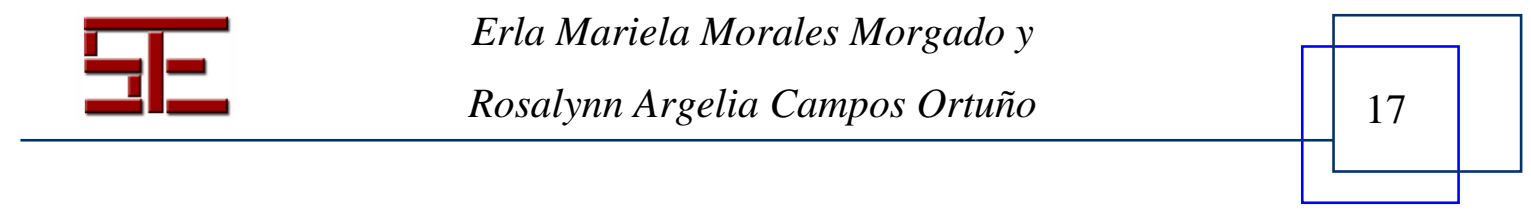




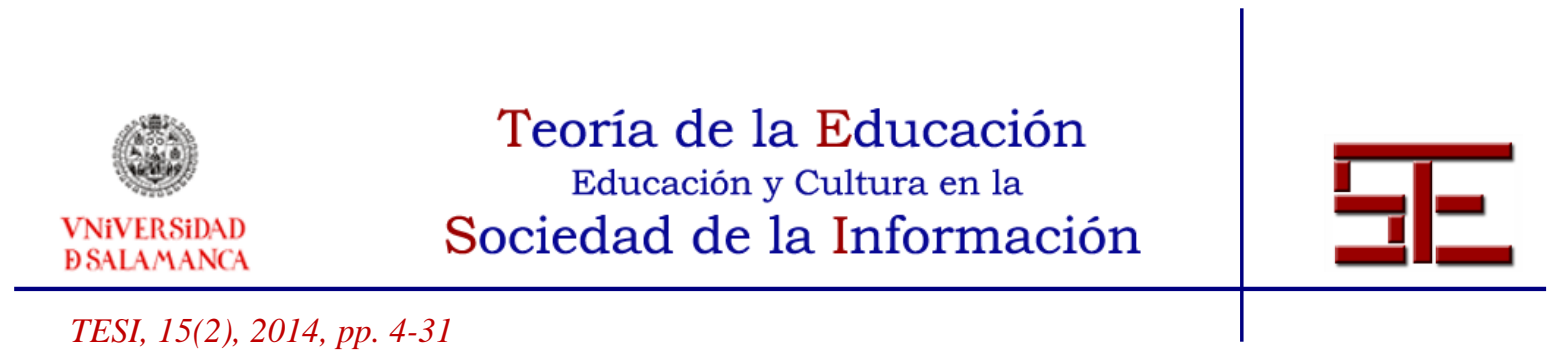

LAS ACTIVIDADES DEL RECURSO EDUCATIVO REVISADO Respuesta

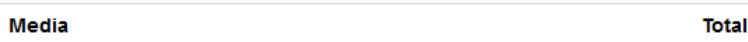

ME INTERESARON POCO

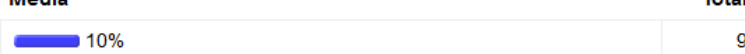

ME INTERESARON BASTANTE

ME INTERESARON MUCHO

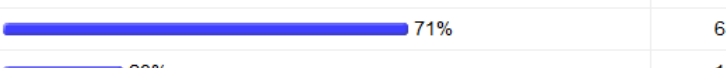

Total

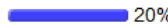

. ¿CONSIDERAS QUE LAS ACTIVIDADES DEL RECURSO EDUCATIVO REVISADO ES ADECUADA?

Respuesta

Media

TOTALMENTE EN DESACUERDO

- $1 \%$

EN DESACUERDO

$2 \%$

DE ACUERDO

TOTALMENTE DE ACUERDO

Total



11. CONSIDERANDO UNA ESCALA DEL 1 AL 4

LAS ACTIVIDADES DEL RECURSO EDUCATIVO SON ADECUADAS EN CUANTO A:

\begin{tabular}{|c|c|c|c|c|c|c|c|}
\hline & & & & Rang & edi & & \\
\hline & & & 1 & 2 & 3 & 4 & \\
\hline & Objetivo & & & & & & 3.1 \\
\hline & Contenido & & & & & & 3.2 \\
\hline & Instrucciones & & & & & & 3.2 \\
\hline & Forma de auto-evaluar & & & & I & & 3.0 \\
\hline & Tiempo para ejecutarla & & & & & & 3.2 \\
\hline & Ejercicios & & & & I & & 3.1 \\
\hline 12. ¿COMPRENDI & STE MEJOR LOS CONTENID & DUCATIVO & & & & & \\
\hline & Respuesta & Media & & & & & Total \\
\hline & EN DESACUERDO & $3 \%$ & & & & & 3 \\
\hline & DE ACUERDO & 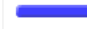 & & & & & 67 \\
\hline & TOTALMENTE DE ACUERDO & 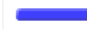 & & & & & 22 \\
\hline & Total & 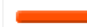 & & & & $00 \%$ & 92/92 \\
\hline
\end{tabular}

Figura 5. Nivel de satisfacción en relación a contenidos y actividades.

VALORA EL RECURSO EDUCATIVO REVISADO

\begin{tabular}{|l|l|r|r|}
\hline Respuesta & Media & Total \\
\hline NO TAN BUENO & $2 \%$ & $82 \%$ & 2 \\
\hline BUENO & & $16 \%$ & 75 \\
\hline MUY BUENO & & $15 \%$ & 15 \\
\hline Total & & $92 / 92$ \\
\hline
\end{tabular}

Figura 6. Valoración general del recurso.

En relación a las actividades realizadas, tal como refleja la figura 5, la evaluación cuantitativa reflejó un alto nivel de aprobación por parte de los estudiantes. En relación al nivel de interés en el recurso revisado un $71 \%$ lo consideró bastante interesante, mientras un $20 \%$ lo consideró de un nivel de interés muy alto.

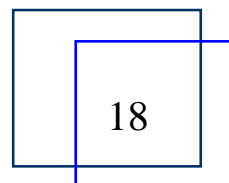

Erla Mariela Morales Morgado y 




El nivel de adecuación de las actividades que presenta el recurso fue altamente valorado, con un $72 \%$ de acuerdo y un $25 \%$ de total acuerdo. El nivel de adecuación fue valorado más específicamente en relación a: objetivos, contenidos, instrucciones, forma de autoevaluar, tiempo de ejecución y ejercicios presentados, aspectos que fueron evaluados en un rango superior a tres, en una escala de valoración de 1 a 4 .

En cuanto a la comprensión de los contenidos del OA, un 73\% manifestó estar de acuerdo, un $24 \%$ total acuerdo, frente a un $3 \%$ en desacuerdo. Finalmente, el $82 \%$ valoró el recurso como bueno, frente al $16 \%$ que lo calificó como muy bueno y un $2 \%$ como no tan bueno.

Para precisar las valoraciones cuantitativas, el cuestionario presentó un campo de texto para explicar el porqué de esa valoración. Según los resultados finales muchos de los estudiantes manifestaron desconocer información detallada sobre los recursos revisados, especialmente en relación al plagio normas APA.

\section{2.- Valoración por parte de docentes}

La valoración de la calidad de los $\mathrm{OA}$ ha sido también validada por un grupo de 4 docentes que participaron en este proyecto de innovación docente. El cuestionario de evaluación aplicado ha sido una adaptación de la Herramienta de Evaluación de Objetos Didácticos de Aprendizaje (HEODAR) (Módulo que se puede instalar en Moodle para evaluar OA SCORM, arrojando de forma inmediata los aspectos mejor y peor evaluados) (Morales et al., 2008). En esta ocasión, se decidió crear un cuestionario basado en los mismos criterios pedagógicos y técnicos que HEODAR, de manera que se pudiesen evaluar los cuatro OA a la vez.

Los aspectos pedagógicos evaluados en su dimensión pedagógica y didácticocurricular y los resultados obtenidos se reflejan en las figuras 7, 8, 9 y 10. Dentro de los aspectos pedagógicos, se observa que el OA sobre plagio académico recibió la puntuación más alta, en los criterios relacionados a: motivación y atención, desempeño profesional, nivel de dificultad adecuado a los destinatarios y creatividad. Según los comentarios de los docentes se puede deducir que el tema en cuestión es uno de los más necesarios a tratar en el contexto universitario. Este recurso recibió una menor puntuación en el criterio que valora la interactividad, a pesar de que cuenta con actividades interactivas, esta interactividad es posiblemente menor que la que presentan los OA con una leve mayor puntuación: buscadores en la Red y buscando en Google.

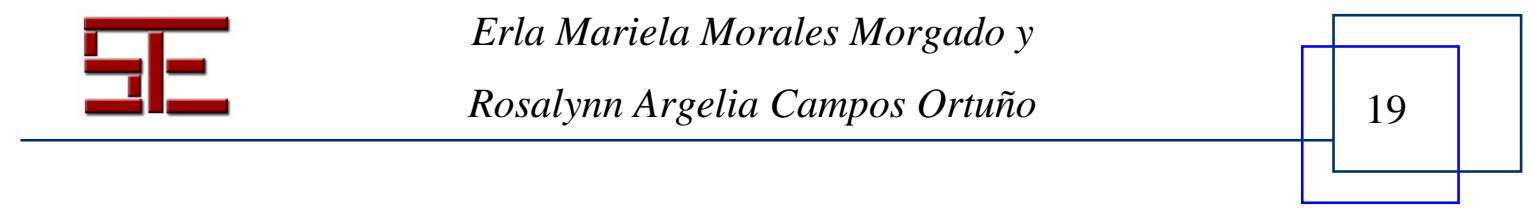




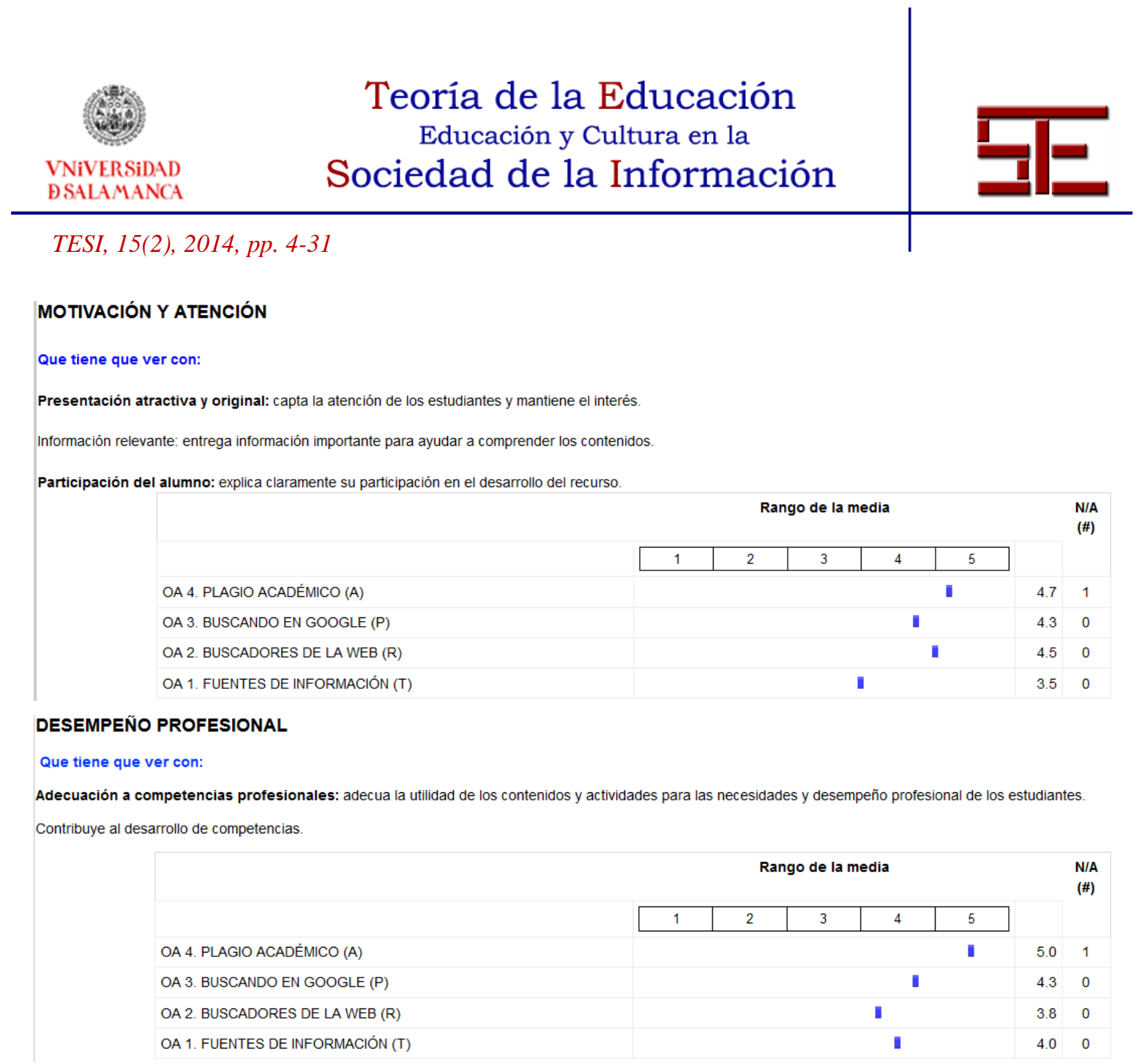

NIVEL DE DIFICULTAD ADECUADO A LAS CARACTERISTICAS DE LOS ESTUDIANTES

Que tiene que ver con:

Profundidad pertinente: adecua la profundidad según conocimientos previos, nivel académico y de complejidad que el estudiante es capaz de comprender

Nivel de Lenguaje: adecua lenguaje utilizado (cientifico, etc.) a los conocimientos previos de los estudiantes o a I nivel académico.

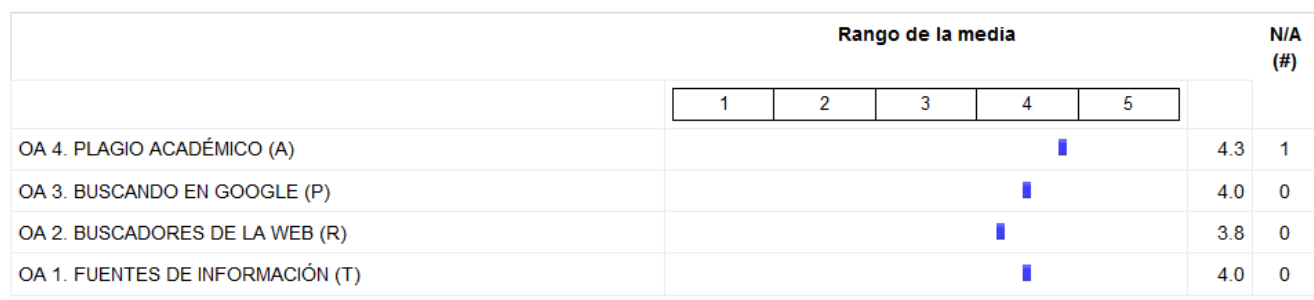

Figura 7. Valoración de aspectos pedagógicos (motivación, desempeño y dificultad).

$200 \begin{aligned} & \text { Erla Mariela Morales Morgado y } \\ & \text { Rosalynn Argelia Campos Ortuño }\end{aligned}$




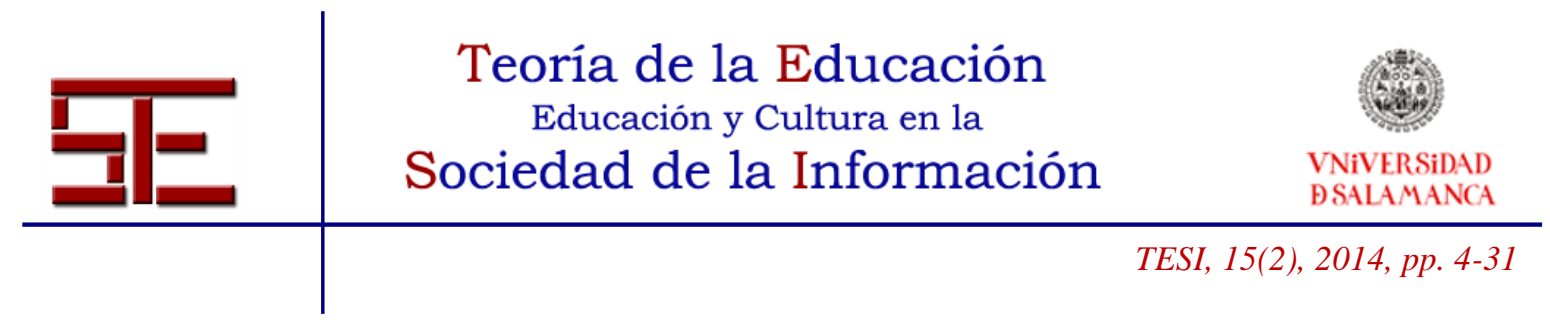

INTERACTIVIDAD

Que tiene que ver con:

Nivel de interactividad: promueve actividades abiertas, diversas maneras de resolver problemas, proporcionar retroalimentación y corrección de errores.

Tipo de interactividad: adecua la interactividad a los objetivos de la metodologia, los niveles pueden ser: activos, expositivos o mixtos.

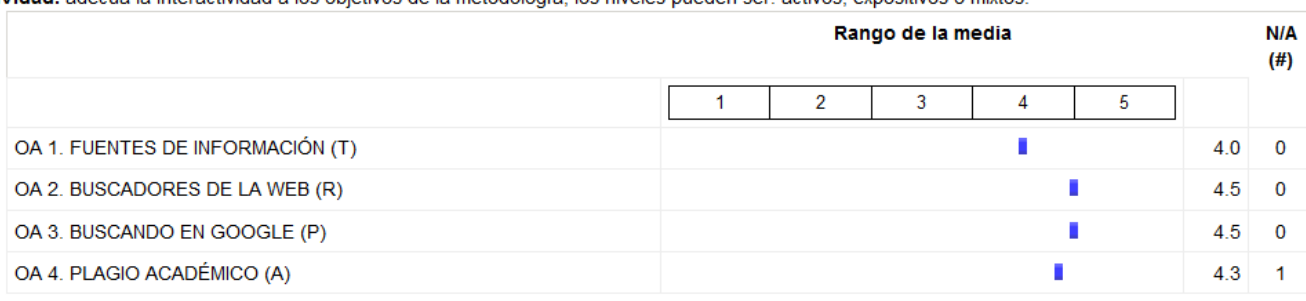

CREATIVIDAD

Que tiene que ver con :

Promueve el desarrollo de iniciativas y del aprendizaje autónomo.

Promueve el desarrollo de habilidades metacognitivas y estrategias de aprendizaje que les permita planificar, regular y evaluar su propia actividad intelectual. Rango de la media

OA 1. FUENTES DE INFORMACIÓN (T)

OA 2. BUSCADORES DE LA WEB (R)

OA 3. BUSCANDO EN GOOGLE (P)

OA 4. PLAGIO ACADÉMICO (A)

\begin{tabular}{|c|c|c|c|c|c|c|}
\hline \multicolumn{6}{|c|}{ Rango de la media } & \multirow{2}{*}{$\begin{array}{l}\text { N/A } \\
(\#)\end{array}$} \\
\hline 1 & 2 & 3 & 4 & 5 & & \\
\hline & \multicolumn{4}{|c|}{ I } & 3.8 & 0 \\
\hline & \multicolumn{4}{|c|}{ I } & 4.0 & 0 \\
\hline & \multicolumn{4}{|c|}{ 【 } & 4.3 & 0 \\
\hline & & & & & 4.7 & 1 \\
\hline
\end{tabular}

Figura 8. Valoración de aspectos pedagógicos (interactividad y creatividad).

En cuanto a los aspectos didáctico-curriculares, representados en la figura 9, el OA sobre plagio académico también cuenta con la mayor puntuación en los criterios. Contexto, objetivos, contenidos, actividades y retroalimentación. La adecuación de contenidos y actividades se muestra bastante pareja en todos los OA, sin embargo, el OA sobre fuentes de información presenta la puntuación más baja en relación al contexto en donde ha sido aplicado y los objetivos de enseñanza, esto puede ser debido a que según los comentarios de los estudiantes los temas tratados en ese recurso eran más conocidos para ellos, sobre los cuales ya tenían algunas nociones.

Sobre los aspectos técnicos sobre diseño de interfaz y navegación, los docentes también señalaron el OA sobre plagio académico con mayor puntuación, sin distar de mucho de los otros OA.

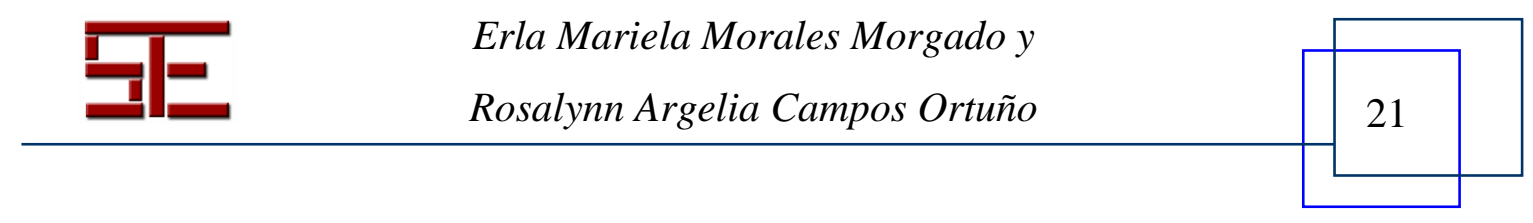




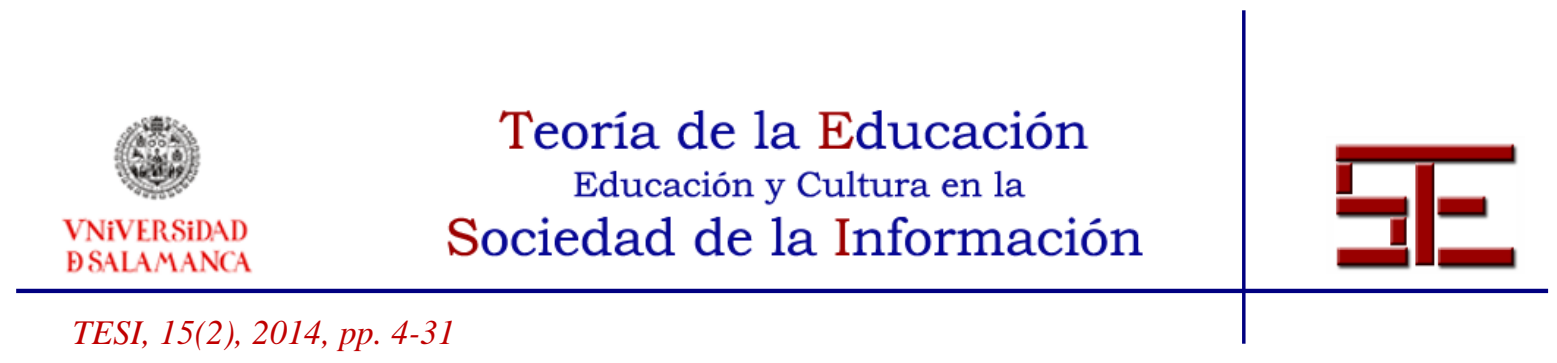

PARTE II. SOBRE LO DIDACTICO-CURRICULAR

11.

CONTEXTO

Que tiene que ver con :

Nivel formativo adecuado a la situación educativa, por ejemplo: educación secundaria, universitario, etc.

\begin{tabular}{|c|c|c|c|c|c|c|c|}
\hline & \multicolumn{5}{|c|}{ Rango de la media } & & \multirow{2}{*}{$\begin{array}{l}\text { N/A } \\
\text { (\#) }\end{array}$} \\
\hline & 1 & 2 & 3 & 4 & 5 & & \\
\hline OA 1. FUENTES DE INFORMACIÓN (T) & & \multicolumn{4}{|c|}{ I } & 3.8 & 0 \\
\hline OA 2. BUSCADORES DE LA WEB (R) & & \multicolumn{4}{|c|}{ I } & 4.3 & 0 \\
\hline OA 3. BUSCANDO EN GOOGLE (P) & & \multicolumn{4}{|c|}{ I } & 4.5 & 0 \\
\hline OA 4. PLAGIO ACADÉMICO (A) & & & & & I & 5.0 & 1 \\
\hline
\end{tabular}

OBJETIVOS

Que tiene que ver con :

Correctamente formulado: generalmente los objetivos se elaboran según la fórmula: verbo infinitivo + contenido +

Factible: puede ser alcanzado

Indica lo que se espera sea aprendido: el alumno debe ser consciente de lo que tiene que aprender.

Coherente con los objetivos generales: los objetivos especificos deben ayudar a cumplir los objetivos generales.

OA 1. FUENTES DE INFORMACIÓN (T)

\begin{tabular}{|l|l|l|l|l|}
\hline 1 & 2 & 3 & 4 & 5 \\
\hline
\end{tabular}

OA 2. BUSCADORES DE LA WEB (R)

OA 3. BUSCANDO EN GOOGLE (P)

【

OA 4. PLAGIO ACADÉMICO (A)

CONTENIDOS

Que tiene que ver con :

Presenta información suficiente y adecuada al nivel educativo.

Adecua los contenidos al objetivo propuesto

Presenta información en distintos formatos (texto, audio, etc)

Permite interactuar con el contenido a través de enlaces.

Presenta información complementaria para ayudar a los alumnos que deseen profundizar sus conocimientos.

Cuida que la información que presenta sea confiable, (datos exactos, referencias bibliográficas, etc.).

Presenta la información de forma adecuada para ayudar a una mejor comprensión del contenido

Verifica que el idioma empleado en los contenidos sea pertinente a los objetivos de enseñanza.

\begin{tabular}{|c|c|c|c|c|c|c|c|}
\hline & \multicolumn{5}{|c|}{ Rango de la media } & & \multirow{2}{*}{$\begin{array}{l}\text { N/A } \\
(\#)\end{array}$} \\
\hline & 1 & 2 & 3 & 4 & 5 & & \\
\hline OA 1. FUENTES DE INFORMACIÓN (T) & & \multicolumn{4}{|c|}{ 【 } & 4.0 & 0 \\
\hline OA 2. BUSCADORES DE LA WEB (R) & & \multicolumn{4}{|c|}{ 【 } & 4.0 & 0 \\
\hline OA 3. BUSCANDO EN GOOGLE (P) & & \multicolumn{4}{|c|}{ ॥ } & 4.3 & 0 \\
\hline OA 4. PLAGIO ACADÉMICO (A) & & & & & I & 5.0 & 1 \\
\hline
\end{tabular}

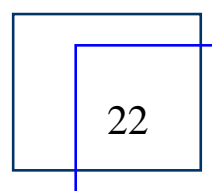

Erla Mariela Morales Morgado y 


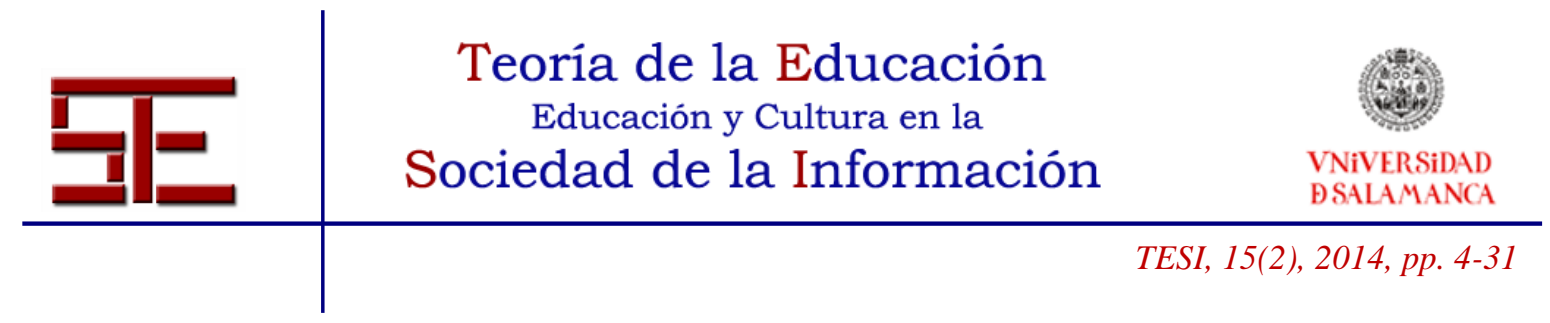

Figura 9. Valoración de aspectos didáctico-curriculares (contexto, objetivos y contenidos).

\section{ACTIVIDADES}

Que tiene que ver con:

Ayudar a reforzar los conceptos

Promueve una participación activa: estimula la reflexión y la crítica, esto es el cuestionamiento de las propias ideas para la integración de la nueva información a los \& pre-existentes

Presenta distintos tipos de estrategias de aprendizaje, según sea el caso (resolución de problemas, estudio de caso)

Presenta actividades de evaluación y práctica

Propone modalidad de trabajo según sea el caso (individual, colaborativa y/o cooperativa)

\begin{tabular}{|c|c|c|c|c|c|c|c|}
\hline & \multicolumn{5}{|c|}{ Rango de la media } & & \multirow{2}{*}{$\begin{array}{l}\text { N/A } \\
(\#)\end{array}$} \\
\hline & 1 & 2 & 3 & 4 & 5 & & \\
\hline OA 1. FUENTES DE INFORMACIÓN (T) & \multicolumn{5}{|c|}{ I } & 3.5 & 0 \\
\hline OA 2. BUSCADORES DE LA WEB (R) & \multicolumn{5}{|c|}{ 【 } & 3.5 & 0 \\
\hline OA 3. BUSCANDO EN GOOGLE (P) & \multicolumn{5}{|c|}{ I } & 3.5 & 0 \\
\hline OA 4. PLAGIO ACADÉMICO (A) & \multicolumn{5}{|c|}{ ! } & 4.3 & 1 \\
\hline
\end{tabular}

\section{RETROALIMENTACION}

Que tiene que ver con:

Se refuerzan los conocimientos a través de ejercicios, autoevaluaciones, etc

Presentan comentarios para afianzar los contenidos.

\begin{tabular}{|c|c|c|c|c|c|c|c|}
\hline & \multicolumn{5}{|c|}{ Rango de la media } & & \multirow{2}{*}{$\begin{array}{l}\mathrm{N} / \mathrm{A} \\
(\#)\end{array}$} \\
\hline & 1 & 2 & 3 & 4 & 5 & & \\
\hline OA 1. FUENTES DE INFORMACIÓN (T) & \multicolumn{5}{|c|}{ 】 } & 3.5 & 0 \\
\hline OA 2. BUSCADORES DE LA WEB (R) & \multicolumn{5}{|c|}{ I } & 3.3 & 0 \\
\hline OA 3. BUSCANDO EN GOOGLE (P) & \multicolumn{5}{|c|}{ 】 } & 3.8 & 0 \\
\hline OA 4. PLAGIO ACADÉMICO (A) & \multicolumn{5}{|c|}{ I } & 4.3 & 1 \\
\hline
\end{tabular}

Figura 10. Valoración de aspectos didáctico-curriculares (actividades y retroalimentación).

\section{5.- CATALOGACIÓN DE LOS OBJETOS DE APRENDIZAJE BASADOS EN COMPETENCIA INFORMACIONAL}

Las dimensiones presentadas para clasificar los OA tienen como finalidad ofrecer también información que permita catalogar los recursos a través de sus metadatos. Los estándares de metadatos actuales como es caso de LOM, Dublin Core, etc., contienen categorías de elementos para agregar información, las cuales no pueden ser

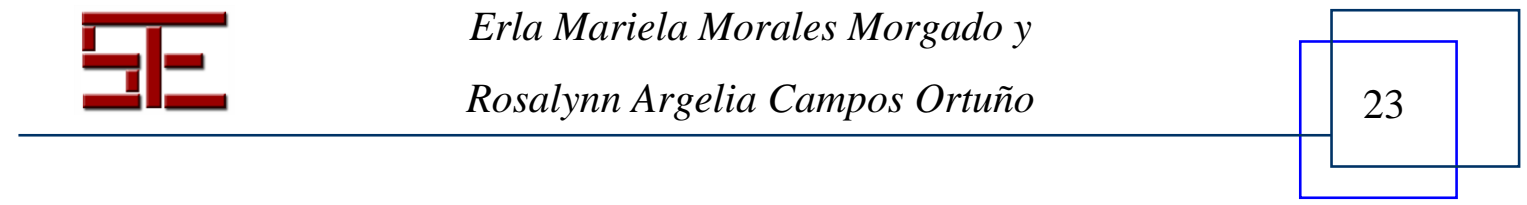




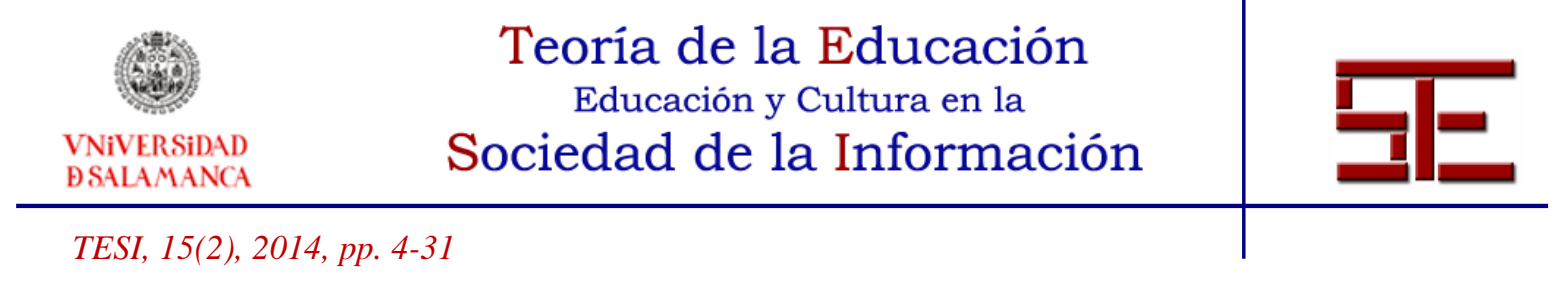

modificadas, por tanto, no es posible agregar elementos de metadatos que hagan referencias a competencias.

Uno de los propósitos del proyecto de innovación en el cual se enmarca esta investigación es difundir los OA a través del repositorio de contenidos institucional de la Universidad de Salamanca GREDOS (gredos.usal.es), de manera que puedan acceder a los recursos todos los miembros de la comunidad universitaria y ser una fuente de consulta para aprender sobre competencia informacional. .

Con este objetivo se ha realizado una propuesta para adaptar los metadatos Dublin Core con el que se catalogan los recursos de GREDOS a través de la plataforma Dspace. (Morales et al., 2013a) (Morales et al., 2013b). La propuesta considera una colección específica para agregar OA llamada "DIRED", la cual contiene una colección para OA en base a competencias y otra en base a estilos de aprendizaje, ésta última basada en una propuesta de Campos Ortuño (2013).

La información de cada una de las dimensiones se presenta a través de una pestaña desplegable, para lo cual se ha agregado la siguiente codificación en XML para la dimensión "4.Competencia Digital", "4.1. Búsqueda, selección, almacenamiento y registro de información", "4.2. Organización, tratamiento y presentación de la información” y “4.3. Comunicación de la Información”.

$<$ ?xml version="1.0" encoding="UTF-8"? >

$<$ node id="COMP_DIGITAL-4" label="4.Competencia Digital">

$<$ isComposedBy>

$<$ node id="COMP_DIGITAL-4.1" label="4.1. Búsqueda, selección, almacenamiento y registro de información" $>$

$<$ isComposedBy>

$<$ node id="COMP_DIGITAL-4.1.1" label="4.1.1. Acceder a la información a

través de herramientas y estrategias específicas" $></$ node $>$

$<$ node id="COMP_DIGITAL-4.1.2"label="4.1.2. Fuentes de

información" $></$ node $>$

$<$ node id="COMP_DIGITAL-4.1.3" label="4.1.3. Valoración crítica y sistemática

de la pertinencia de la información" $>\langle$ /node $>$

$</$ isComposedBy>

Código XML para la dimensión "4.1. Búsqueda, selección, almacenamiento y registro de información".

<node id="COMP_DIGITAL-4.2" label="4.2. Organización, tratamiento y

presentación de la información">

$<$ isComposedBy>

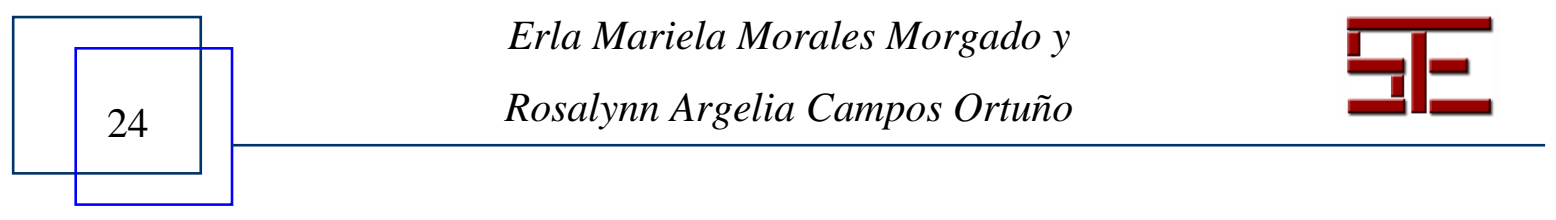




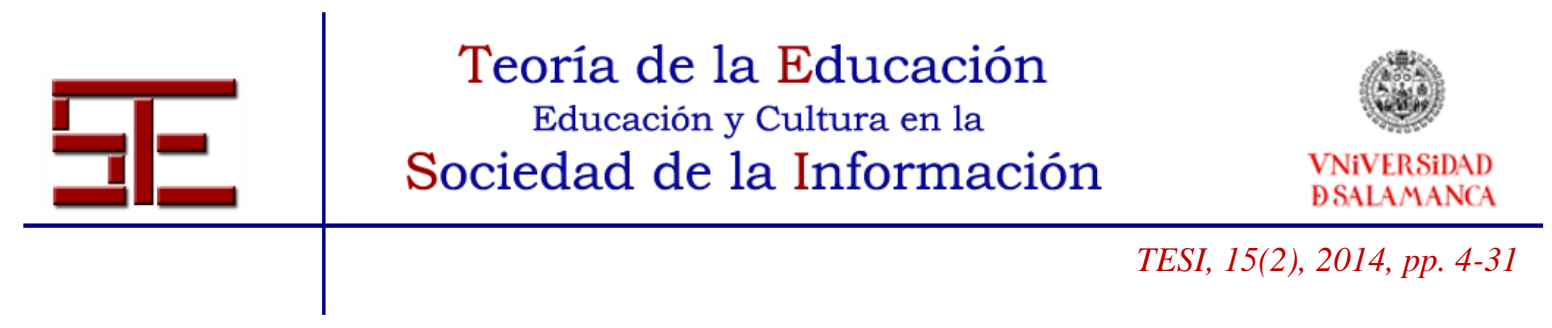

<node id="COMP_DIGITAL-4.2.1" label="4.2.1. Almacenamiento y registro de la información en diversas fuentes" $></$ node $>$

<node id="COMP_DIGITAL-4.2.2" label="4.2.2. Manejar diversos tipos y códigos de información: textual, numérica, icónica, gráfica, auditiva y audiovisual" $></$ node $>$

$<$ node id="COMP_DIGITAL-4.2.3" label="4.2.3. Manejar herramientas para

producir, presentar y comprender información" $\rangle\langle/$ node $\rangle$

$<$ node id="COMP_DIGITAL-4.2.4" label="4.2.4. Intercambiar

información" $></$ node $>$

$</$ isComposedBy $>$

Código XML para la dimensión “4.2. Búsqueda, selección, almacenamiento y registro de información"

$<$ node id="COMP_DIGITAL-4.3" label="4.3. Comunicación de la información" $\rangle\langle/$ node $\rangle$

$<$ isComposedBy $>$

$<$ node id="COMP_DIGITAL-4.3.1" label="4.3.1. Herramientas para compartir y trabajar en colaboración" $></$ node $>$

$<$ node id="COMP_DIGITAL-4.3.2" label="4.3.2. Difusión de la información en diversos soportes" $></$ node $>$

$<$ node id="COMP_DIGITAL-4.3.3" label="4.3.3. Desarrollo del pensamiento

crítico, creatividad e innovación" $></$ node $>$

$</$ isComposedBy>

Código XML para la dimensión “4.3. Comunicación de la información”.

La finalidad de esta clasificación es que los OA puedan ser almacenados y recuperados según las necesidades específicas de formación en competencias informacionales, aportando información básica sobre los criterios, descriptores y habilidades y destrezas a desarrollar.

\section{6.- CONCLUSIONES}

Como se puede deducir en el primer apartado, la necesidad de formar a los estudiantes universitarios en competencias informacionales debe ser una prioridad de la institución educativa, lo cual requiere el planteamiento de una estrategia que contemple idealmente planes formativos complementarios a la formación reglada, con la colaboración de personal especializado en bibliotecas, que orienten a los estudiantes a saca el máximo provecho de las herramientas, recursos, repositorios y bases de datos, algunas sobre las

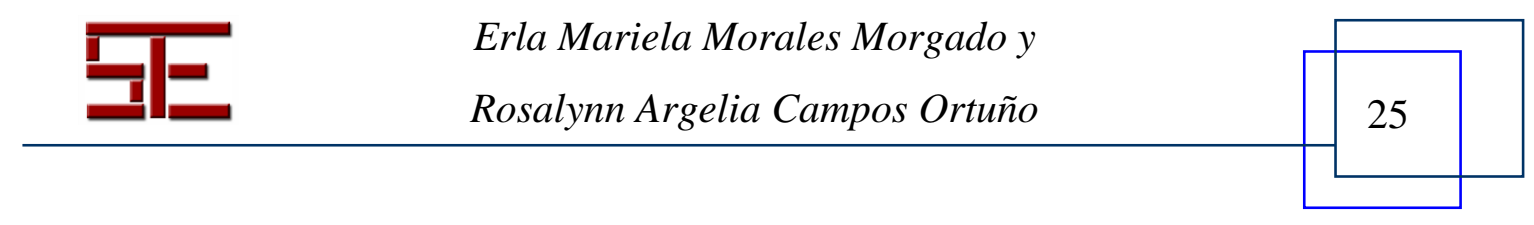




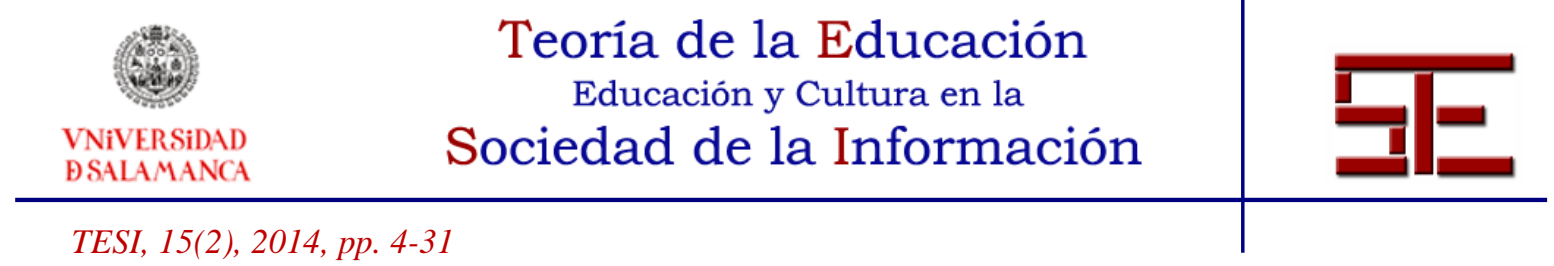

cuales la Universidad invierte dinero para el acceso de los miembros de la comunidad Universitaria.

Dentro de los planes formativos, la presente propuesta pretende contribuir con materiales específicos al desarrollo de la competencia informacional, apuntando a objetivos concretos de aprendizaje, con los contenidos y actividades necesarios para su logro a su vez se destaca el diseño de los contenidos en base a estilos de aprendizaje, de manera que ayuden a mejorar la comprensión de estudiantes que tengan diversas necesidades de formación.

Los resultados obtenidos en las valoraciones por parte de estudiantes reflejan una gran acogida en relación a los contenidos y actividades ofertadas, lo cual queda evidenciado en los comentarios que éstos realizan sobre su nivel de satisfacción en relación a la utilidad de los conocimientos ofrecidos para ayudarles a mejorar sus competencias informacionales.

La evaluación por parte de docentes también refleja un alto nivel de satisfacción con respecto a los recursos, valorando de forma más alta el contenido y actividades relacionado a plagio académico y normas APA, ya que es uno de los temas que presenta mayor dificultad entre los estudiantes, lo cual se refleja de manera continua en los trabajos que éstos deben presentar a través de las diversas asignaturas y trabajos finales de grado.

A través de la valoración cualitativa los docentes manifestaron que se deben realizar algunas mejoras en relación a la claridad de la información presentada, aspectos que serán considerados en la mejora de los recursos antes de su publicación en el repositorio institucional GREDOS.

La adaptación de metadatos para almacenar los OA en GREDOS es otro valor añadido de esta propuesta, la cual pretende promover el uso del repositorio institucional y el acceso a los recursos por parte de cualquier miembro de la comunidad universitaria, lo cual permitirá ser utilizado como una referencia para docentes y estudiantes de cualquier titulación. Por otra parte, esta propuesta de metadatos puede ser aplicada a repositorios de Objetos de Aprendizaje a través de la reutilización de los metadatos de una colección digital (López et al., 2005) y arquitecturas para la recuperación de OA en repositorios distribuidos (Morales et al., 2007a).

Los metadatos mencionados en este artículo para la competencia informacional permitirán una rápida identificación de los objetivos, habilidades y destrezas informacionales que se promueven, lo cual ayudará a los usuarios a seleccionar y recuperar el recurso que más se adecue a sus necesidades formativas.

La competencia informacional es solo una las competencias que los estudiantes universitarios deben desarrollar. Esta iniciativa pretende incentivar a otros docentes para que elaboren y divulguen otros materiales, ya sea para competencias digitales $u$ otras

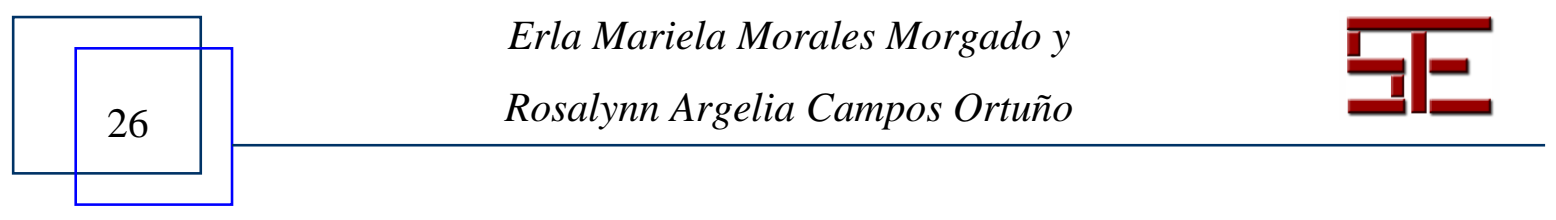




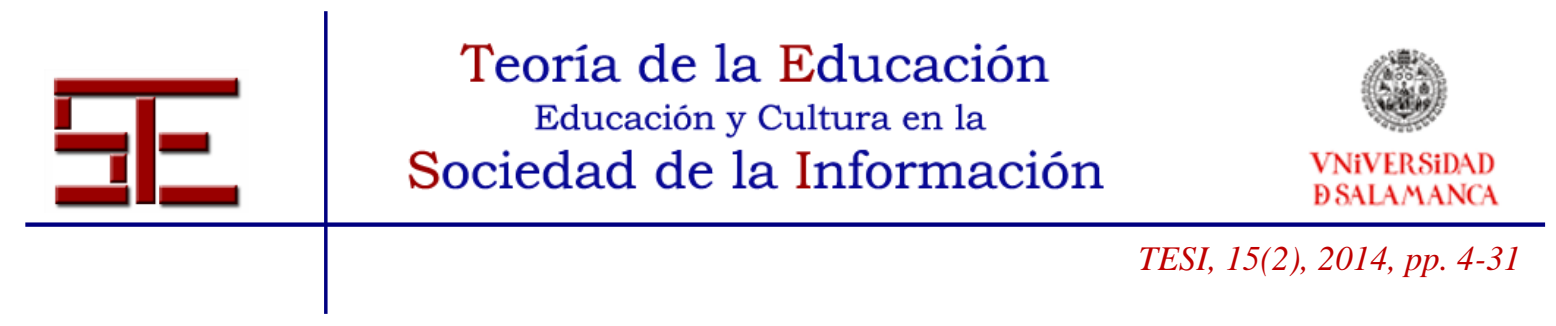

relacionadas al desempeño profesional. El uso del repositorio institucional, al ser de acceso abierto, facilita la recolección de los recursos por otros buscadores de índole nacional e internacional, por tanto, es una estupenda alternativa para que los docentes puedan difundir sus materiales y experiencias de aula. Finalmente, esta propuesta puede ser parte de un sistema de gestión de Objetos de Aprendizaje de Calidad a nivel institucional, que considere la evaluación de los recursos en diversos momentos de su gestión (Morales, 2010).

\section{7.- BIBLIOGRAFÍA}

Area Moreira, M. (2007). Adquisición de competencias en información. Una materia necesaria en la formación universitaria. Documento marco REBIUN para la CRUE [versión electrónica]. http://www.rebiun.org/doc/adquisicion\%20de\%20 competencias.doc.

Area Moreira, M. (2010). ¿Por qué formar en competencias informacionales y digitales en la educación superior? Revista de Universidad y Sociedad del Conocimiento, 7 (2), 2-5. Recuperado a partir de http://www.uoc.edu/ojs/index.php/rusc/article/viewFile/v7n2-area/v7n2-area.

Campos Ortuño, R., \& Morales Morgado, E. M. (2013). Influence of Learning Objects based on Learning Styles. En Actas del III Congreso Ibérico de Innovación en Educación con las TIC. Celebrado los días 17, 18 y 19 de octubre en la Facultad de Educación de la Universidad de Salamanca. En prensa.

Castells, M. (1999). La era de la información: economía, sociedad y cultura. La sociedad Red (vol. 1). Madrid: Alianza Editorial.

CNICE. (2007). Las TIC en la Educación: panorama internacional y situación española. Madrid: Santillana. Recuperado a partir de http://www.oei.es/tic/DocumentoBasico.pdf.

COMMISSION OF THE EUROPEAN COMMUNITIES. (2000). A Memorandum on Lifelong Learning. MECD. Recuperado a partir de http://ec.europa.eu/education/lifelong-learning-policy/doc/policy/memo_es.pdf

CRUE-TIC \& REBIUN. (2012). Competencias informáticas e informacionales en los estudios de grado [versión electrónica]. [Fecha de consulta: 02/05/14]. http://ci2.es/sites/default/files/documentacion/ci2_estudios_grado.pdf.

CRUE-TIC \& REBIUN. (2013). Manual para la formación en competencias informáticas e informacionales (CI2). Recuperado a partir de http://ci2.es/sites/default/files/documentacion/manual_ci2_completo.pdf.

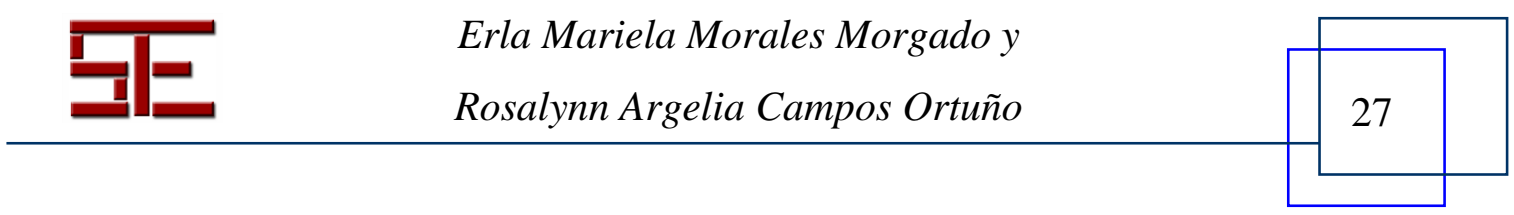




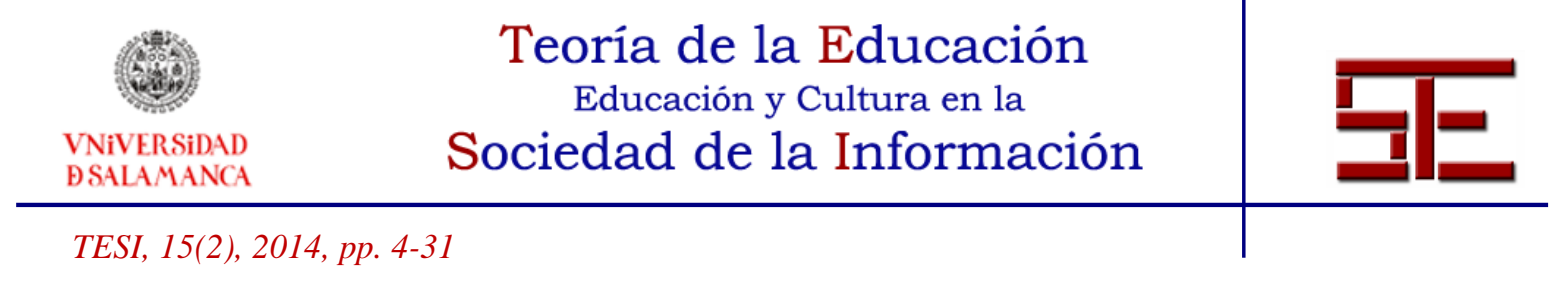

Diario oficial de la Unión Europea. (2006). Recomendación del Parlamento Europeo y del Consejo de 18 de diciembre de 2006 sobre las competencias clave para el aprendizaje permanente. Bruselas: Parlamento Europeo y Consejo de la Unión europea. Recuperado a partir de http://eur-lex.europa.eu/LexUriServ/site/es/oj/ 2006/1_394/1_39420061230es00100018.pdf.

Duque Pérez, C., Morales Luis, A., Penín Navascués, M. L., \& Penín Navascués, N. (2013). Adivina quién: Tratamiento de la información y competencia digital: la lectura digital. Cuadernos para aprender y comunicar. Editorial, Octaedro Solaris Multimedia.

España. Real Decreto-ley 1513/2006, de 7 de diciembre, por el que se establecen las enseñanzas mínimas correspondientes a la Educación Primaria. Ministerio de la Presidencia, Madrid.

España. Real Decreto-ley 1631/2006, de 29 de diciembre, por el que se establecen las enseñanzas mínimas correspondientes a la Educación Secundaria Obligatoria. BOE, 5, 677-773.

Gaio Alves, M., Neves, C. \& Gomes, E. X. (2010). Lifelong learning: Conceptualizations in European educational policy documents. European Educational Research Journal, 9 (3), 332-344.

Gómez Hernández, J. A. (2002). Prácticas y experiencias de alfabetización informacional en universidades españolas [versión electrónica]. [Fecha de consulta: 01/05/14]. http://www.um.es/fccd/jagh/alfaunivercaceres.PDF.

Gómez Hernández, J. A. \& Benito Morales, F. (2001). De la formación de usuarios a la alfabetización informacional: propuestas para enseñar habilidades de información [versión electrónica]. [Fecha de consulta: 09/05/14]. http://ibersid.eu/ojs/index.php/scire/article/view/1150

González Briones, E. (2011). Alfabetización mediática y competencias básicas. Madrid: Ministerio de Educación, Subdireccion General de Documentación y Publicaciones.

González Teruel, A. (2011). La perspectiva del usuario y del sistema en la investigación sobre el comportamiento informacional. Teoría de la Educación: Educación y Cultura en la Sociedad de la Información, 12 (1), 28-46. Recuperado a partir de http://eprints.rclis.org/bitstream/10760/15535/1/Para\%20elis.pdf

Grupo ALFIN/REBIUN (2008). Guía de buenas prácticas para el desarrollo de las competencias informacionales en las universidades españolas [versión electrónica]. [Fecha de consulta: 03/05/14]. http://www.rebiun.org/export/docReb/guia_buenas_practicas.doc.

$28 \mathrm{E} \quad \begin{aligned} & \text { Erla Mariela Morales Morgado y } \\ & \text { Rosalynn Argelia Campos Ortuño }\end{aligned}$




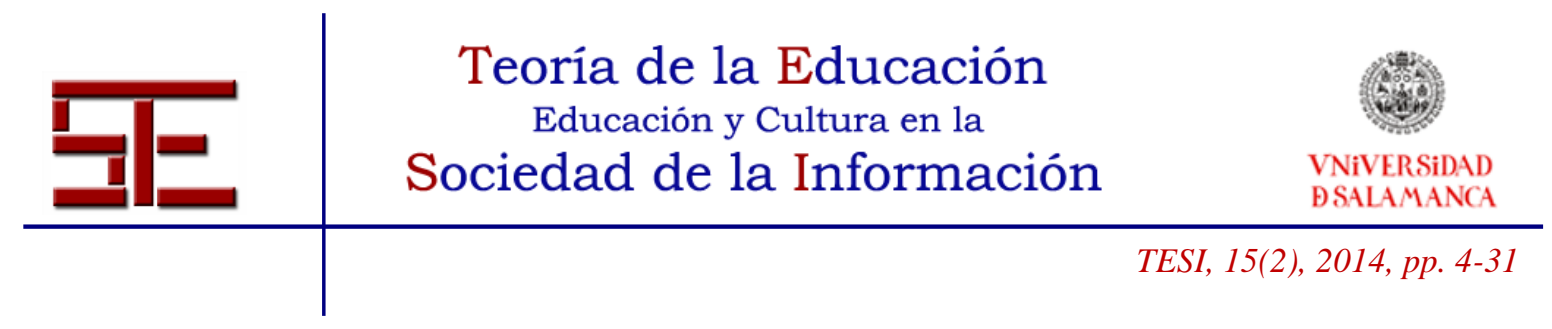

Hernández Hernández, C. J. (2009a). La experiencia de la biblioteca de la Universidad de La Laguna en ALFIN [artículo en línea]. En Seminario: Biblioteca, aprendizaje y ciudadanía. Vilanova i la Geltrú. [Fecha de consulta: 20/04/14]. http://www.alfared.org/content/veintitantas-experiencias-alfin-y-una-canci-nesperanzada/experiencias-alfin-en-bibliotec-20

Hernández Hernández, C. J. (2009b). El programa de formación en competencias informacionales de la biblioteca de la ULL [versión electrónica]. En VII Jornadas CRAI. Competencias informacionales e informáticas en el ámbito universitario. Madrid. [Fecha de consulta: 02/05/14]. http://www.rebiun.org/documentos/Documents/VIIJCRAI/VIICRAI2009_Ponencia_ ProgramaFormacionCompetenciasInformacionales_ULL_CJHernandez.pdf.

Hernández Serrano, M. J. \& Fuentes Agustí, M. (2011). Aprender a informarse en la red: ¿Son los estudiantes eficientes buscando y seleccionando la información? Teoría de la Educación: Educación y Cultura en la Sociedad de la Información , 12 (1), 47-78. Recuperado a partir de http://campus.usal.es/ revistas_trabajo/index.php/ revistatesi/article/view/7823/7850.

López, C., García, F., Pernías, P. (2005). Desarrollo de Repositorios de Objetos de Aprendizaje a través de la Reutilización de los Metadatos de una Colección Digital: De Dublin Core a IMS. RED. Revista de Educación a Distancia. IV, Número monográfico II. 20 de Febrero de 2005.

Morales Morgado, E. M., Campos Ortuño, R. A., Yang, L. \& Ferréras Fernández, T. (2014). Adaptation of Descriptive Metadata for Managing Educational Resources in the GREDOS Repository. International Journal of Knowledge Management (IJKM). In press.

Morales Morgado, E. M., Campos Ortuño, R.A., Yang, L. \& Ferréras Fernández, T. (2013a). Metadata Mapping to describe Learning Objects and educational Apps in the Gredos Repository. Technological Ecosystems for Enhancing Multiculturality Conference, TEEM '13, Salamanca, Spain, November 14-15, 2013. ACM 2013 ISBN 978-1-4503-2345-1: 349-356

Morales Morgado, E. M., Campos Ortuño, R.A., Yang, L. \& Ferréras Fernández, T. (2013b). Proyecto DIRED: Propuesta para divulgar y gestionar recursos educativos a través del repositorio GREDOS. En Actas del III Congreso Ibérico de Innovación en Educación con las TIC. Celebrado los días 17, 18 y 19 de Octubre en la Facultad de Educación de la Universidad de Salamanca. En prensa.

Morales Morgado, E. M., Díaz San Millán, E., García Peñalvo, F. J. (2011). Gestión de objetos de aprendizaje a través de la red, basada en el desarrollo de competencias. Teoría de la Educación. Educación y Cultura en la Sociedad de la Información. 12(1):99-115. Abril 2011

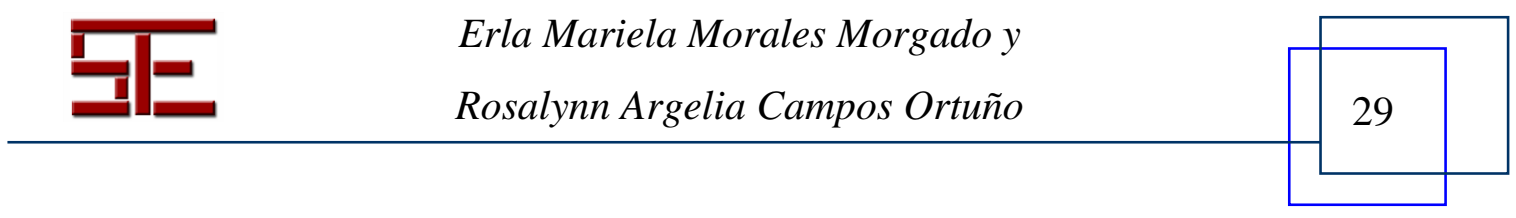




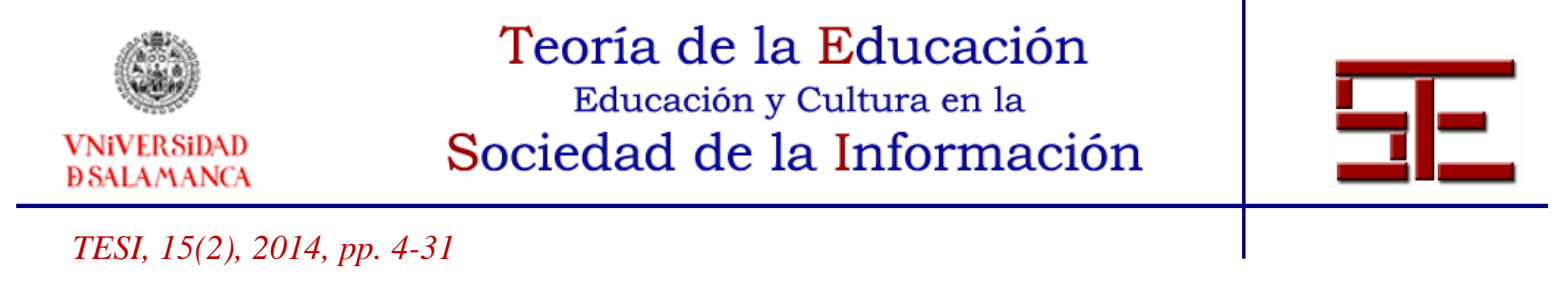

Morales Morgado, E. M. (2010). Gestión del Conocimiento en Sistemas elearning, Basado en Objetos de Aprendizaje Cualitativa y Pedagógicamente definidos. Colección Vítor 273. Ediciones Universidad de Salamanca y Erla Mariela Morales Morgado. I.S.B.N: 978-84-7800-174-3. Depósito legal: S.1.152-2010

Morales Morgado, E. M., Gómez, D. y García Peñalvo, F. J. (2008). HEODAR: Herramienta para la Evaluación de Objetos Didácticos de Aprendizaje Reutilizables. Actas del X Simposio Internacional de Informática Educativa (SIIE'08). J. ÁngelVelázquez Iturbide, Francisco José García Peñalvo y Ana Belén Gil González (Eds.) Colección Aquilafuente, ediciones Universidad Salamanca, 1 al 3 de Octubre del 2008. ISBN: 978-84-7800-312-9

Morales Morgado, E.M., Gil, A. B., García, F. J. (2007a). Arquitectura para la Recuperación de Objetos de Aprendizaje de Calidad en Repositorios Distribuidos. En las Actas del $5^{\circ}$ taller en Sistemas Hipermedia Colaborativos y Adaptativos, SHCA 2007. (Zaragoza, 11 de septiembre de 2007). Celebrado en el Marco de la II Conferencia Española de Informática (CEDI 2007). F. L. Gutiérrez Vela, P. Paderewski Rodriguez (Eds.). Páginas 31-38. Actas de Talleres de Ingeniería del Software y Bases de Datos, SISTEDES, Volumen 1, Número 1.

Morales Morgado, E. M., García, F. J., Barrón, Á. (2007b). Improving LO Quality through Instructional Design Based on an Ontological Model and Metadata. Journal of Universal Computer Science, 13(7):970-979. July 2007.

Morales Morgado, E. M., García, F. J., Barrón, Á. Berlanga, A. J., López, C. (2005). Propuesta de Evaluación de Objetos de Aprendizaje. En las Actas del II Simposio Pluridisciplinar sobre Diseño, Evaluación y Descripción de Contenidos Educativos Reutilizables, SPDECE'05 (Barcelona, 19, 20 y 21 de Octubre de 2005).

Morales Morgado, E. M., García, F. J., Moreira, T., Rego, H., Berlanga, A. (2004). Units of Learning Quality Evaluation. En SPDECE 2004 Design, Evaluation and Description of Reusable Learning Contents. Proceedings of the First PluriDisciplinary Symposium on Design, Evaluation and Description of Reusable Learning Contents. (Guadalajara (Spain), October 20-22, 2004). J. R. Hilera González, J. A. Gutiérrez de Mesa, R. Vélez de Miguel, R. Martínez Borda (Eds.). CEUR Workshop Proceedings Vol. 117. http://ceur-ws.org/Vol-117

Muñoz, C., Conde, M. Á., García, F. J. (2010a). Moodle HEODAR implementation and its implantation in an academic context. International Journal of Technology Enhanced Learning (IJTEL), 2(3):241-255. Inderscience.

Muñoz, C., García Peñalvo, F. J., Morales, E. M., Conde, M. Á., Seoane, A. M. (2010b). Improving Learning Object Quality: Moodle HEODAR Implementation. International Journal of Distance Education Technologies (IJDET), 10(4): 1-16, October-December 2012. DOI: 10.4018/jdet.2012100101

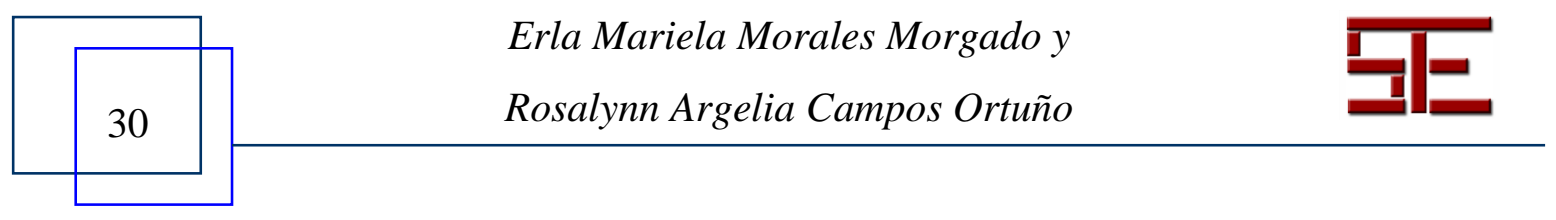




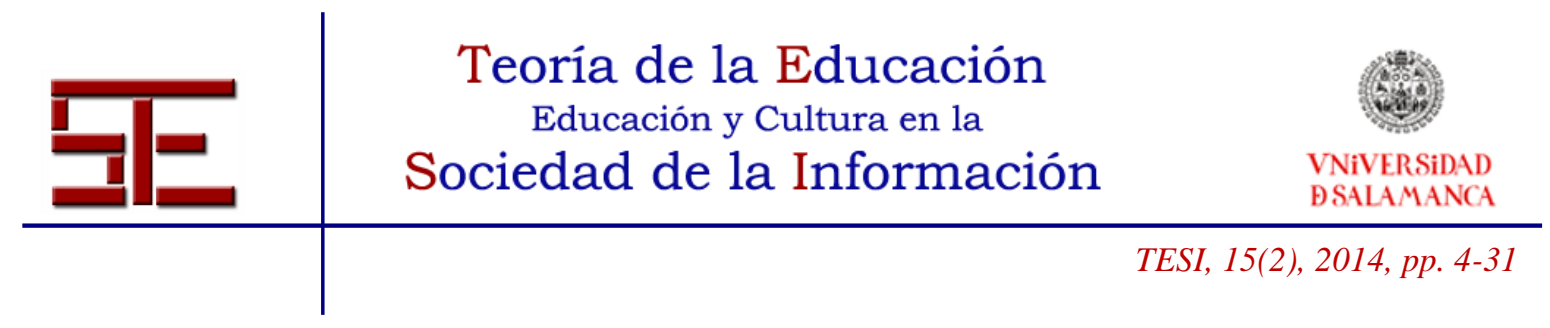

Universidad de Sevilla. Biblioteca. (2009). Las competencias informacionales (CI) en las titulaciones de grado y postgrado de la Universidad de Sevilla. Propuesta de integración [versión electrónica]. $<$ http://formacionbus.pbworks.com/f/Propuesta\%2B Integracion\%2BALFIN\%2Ben\%2BTitulaciones-1. doc>

Universitat Politècnica de Catalunya. Servei de Biblioteques I Documentació. (2007). Proposta d'integració i formació de la competència transversal en Habilitats Informacionals (HI) a les titulacions dels estudis de grau i postgrau de la UPC. Document de treball intern. Barcelona: UPC. UNIVERSITAT

Vivancos, J. (2008). Tratamiento de la información y competencia digital. Alianza Editorial.

Para citar el presente artículo puede utilizar la siguiente referencia:

Morales Morgado, E. M. y Campos Ortuño, R. A. (2014). Dimensiones para el diseño y catalogación de objetos de aprendizaje en base a competencias informacionales. Revista Teoría de la Educación: Educación y Cultura en la Sociedad de la Información. 15(2), 4-31 [Fecha de consulta: dd/mm/aaaa].

http://campus.usal.es/ revistas_trabajo/index.php/revistatesi/article/view/11884

Erla Mariela Morales Morgado y

Rosalynn Argelia Campos Ortuño 